\title{
Dynamical correlations and quantum phase transition in the quantum Potts model
}

\author{
Ákos Rapp ${ }^{1,2}$ and Gergely Zaránd ${ }^{1,2}$ \\ ${ }^{1}$ Institut für Theoretische Festkörperphysik, Universität Karlsruhe,76128 Karlsruhe, Germany \\ ${ }^{2}$ Theoretical Physics Department, Institute of Physics, \\ Technical University Budapest,Budapest, H-1521, Hungary
}

(Dated: February 2, 2008)

\begin{abstract}
We present a detailed study of the finite temperature dynamical properties of the quantum Potts model in one dimension. Quasiparticle excitations in this model have internal quantum numbers, and their scattering matrix deep in the gapped phases is shown to take a simple exchange form in the perturbative regimes. The finite temperature correlation functions in the quantum critical regime are determined using conformal invariance, while far from the quantum critical point we compute the decay functions analytically within a semiclassical approach of Sachdev and Damle [K. Damle and S. Sachdev, Phys. Rev. B 57, 8307 (1998)]. As a consequence, decay functions exhibit a diffusive character. We also provide robust arguments that our semiclassical analysis carries over to very low temperatures even in the vicinity of the quantum phase transition. Our results are also relevant for quantum rotor models, antiferromagnetic chains, and some spin ladder systems.
\end{abstract}

PACS numbers: 05.30.-d,05.50.+q,73.43.Nq

\section{INTRODUCTION}

Quantum critical systems have been in the focus of intense studies in the last two decades $\frac{1,2}{2}$ In these systems a second order phase transition driven by quantum fluctuations takes place at $T=0$ temperature, which, nevertheless, determines the physical properties of the system over a finite parameter range, and leads to anomalous dynamical scaling. Much of our interest in these quantum critical systems has been motivated by the anomalous scaling behavior that can be observed in various heavy fermion compounds, 3.4 .5 .6 but the anomalous behavior of the normal states of cuprates has also been interpreted in terms of an underlying quantum phase transition, possibly hidden by the d-wave superconducting state, similar to many heavy fermion compounds 7.8 .9 Much of the studies of quantum critical phase transitions focused on the simplest case of magnetic phase transitions, 1.10,11.12 where typically quantum fluctuations compete with a tendency of magnetic ordering. While in many cases a quantumclassical mapping enables one to map out the phase diagram and determine critical exponents based upon our knowledge and experience with classical criticality, computing real time response functions represents a major challenge for theorists. In this regard 1+1-dimensional systems are of crucial importance since powerful methods can be used there to analyze their dynamical properties both in the gapped phases, $, 2,13,14$ and in the quantum critical regime $\frac{1}{1}$. These one-dimensional models, besides

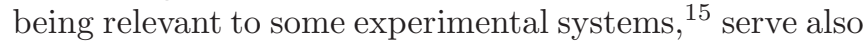
as test grounds for higher-dimensional systems. 16

Maybe the simplest and most thoroughly studied 1+1dimensional model is the transverse field Ising model, where a one-dimensional Ising chain with ferromagnetic coupling $J$ is coupled to a magnetic field $h$ in the $x$ direction. In this model a quantum phase transition takes place from a ferromagnetically ordered to a paramagnetic state at a critical value of the magnetic field, $h_{c} / J=1$, and the critical theory is simply that of the two-dimensional classical Ising model 1.17.18 In this model, dynamical properties are well-understood both in the critical regime and in the gapped phases. ${ }^{1.12}$

In case of the transverse field Ising model, our deep understanding of the quantum critical regime is based on the observation that a 1+1-dimensional critical system is conformally invariant, and one can therefore describe it by means of some conformal field theory ${ }^{18}$ More interestingly, however, we can turn this observation backwards by saying that any conformal field theory should correspond to some model that displays a quantum phase transition.

In this way, we can, in principle classify, construct, and study new one-dimensional quantum mechanical systems which belong to different universality classes, and thus exhibit new and interesting critical behavior.

Relying on this observation, in the present paper we shall study the simplest possible $1+1$-dimensional generalization of the transverse field Ising model which belongs to a different universality class, the $Q$-state quantum Potts model. In this one-dimensional model each spin has $Q$ different components corresponding to the basis states $|\mu\rangle$ with $\mu=1, \ldots, Q$, and the Hamiltonian takes on the following simple form

$$
H=-j \sum_{i} \sum_{\mu} P_{i}^{\mu} P_{i+1}^{\mu}-j g \sum_{i} P_{i}
$$

where the operator $P^{\mu}$ projects on state $|\mu\rangle$ within the $Q$-dimensional local Hilbert space, while $P$ projects to the 'corner' state $\sum_{\mu}|\mu\rangle / \sqrt{Q}$. The ferromagnetic coupling $j$ thus tries to polarize all Potts spins at each site to one of the $Q$ possible orthogonal directions, while the magnetic field $h=j g$ tries to project each spin to the $(1,1, \ldots)$ direction and thus to destroy long range order generated by the ferromagnetic coupling. In the particular case of $Q=2 \mathrm{Eq}$. (1) reduces to the Ising 
Hamiltonian, with $\sum_{\mu=1,2} P_{i}^{\mu} P_{i+1}^{\mu} \equiv\left(\sigma_{i}^{z} \sigma_{i+1}^{z}+1\right) / 2$ and $P_{i} \equiv\left(\sigma_{i}^{x}+1\right) / 2$. For later convenience, we shift the ground state energy and replace the projectors in Eq. (1) by the traceless operators $\tilde{P}^{\mu}=P^{\mu}-\frac{1}{Q}$ and $\tilde{P}=P-\frac{1}{Q}$.

The quantum Potts model defined by Eq. (1) and the corresponding two-dimensional classical Potts model have been studied extensively before, $18,19,20,21,22,23$ However, while we know a lot about the quantum Potts model's thermodynamic behavior, much less is known about its real time dynamics, especially at finite temperatures. The purpose of the present paper is to study in detail dynamical correlations within the quantum Potts model at finite temperatures, both in the gapped and in the quantum critical regimes. As we shall see, many of our results also apply to other one-dimensional gapped systems and are therefore of direct physical relevance.

The one-dimensional quantum Potts model, Eq. (1), is known to exhibit a phase transition as a function of the coupling $g^{22.23}$ : Below a critical value, $g<g_{c}$ the ground state of Eq. (11) is a $Q$-fold degenerate ferromagnet corresponding to the $Q$ different ferromagnetic alignments of the Potts spins. The elementary excitations are domain walls that move along the chain with a dispersion

$$
\epsilon_{k}^{\mu, \mu^{\prime}}=\epsilon(k)
$$

where the two indices $\mu$ and $\mu^{\prime}$ denote the orientations of the ferromagnetic order parameters on the two sides of the excitation, and $k$ is its lattice momentum. These quasiparticles are gapped and their energy can be approximated for small $g$-s as $\epsilon(k) \approx j\left(1-g \frac{2}{Q} \cos k\right)$.

For $g>g_{c}$, on the other hand, the ground state is a non-degenerate paramagnet, which corresponds to orienting all spins along the $(1, \ldots, 1)$ direction. Elementary excitations of this state consist of $\lambda=1, \ldots, Q-1$ possible local 'spin flips', which propagate along the chain with a dispersion

$$
\epsilon_{k}^{\lambda}=\tilde{\epsilon}(k)
$$

These excitations are also gapped and for very large values of $g$ one finds $\tilde{\epsilon}(k) \approx j g\left(1-\frac{2}{Q} \frac{1}{g} \cos k\right)$ from perturbation theory.

Clearly, since the structure of the ground state and its elementary excitations is entirely different for $g \ll 1$ and $g \gg 1$, a phase transition must occur at some critical value $g=g_{c}$. This phase transition turns out to be of second order for $Q \leq 4$, while it is of first order for $Q>4^{20,24}$. This implies that the quasiparticle gap remains finite for $Q>4$, while it approaches zero at $g=g_{c}$ for $Q \leq 4$. The case $Q=4$ is special, and shall not be discussed here: Although the phase transition is continuous for $Q=4$ too, there a marginal operator dominates the critical point ${ }^{25}$ The case $Q=3$, on the other hand, is of special interest: For $Q=3$ one finds a standard second order quantum phase transition, which belongs to a universality class different from the $Q=2$ Ising case 18 . Furthermore, for $Q \geq 3$ the quasiparticles have internal quantum numbers making their dynamics very interesting, and in many ways similar to that of quantum rotor models and $S=1$ spin antiferromagnetic chains ${ }^{1.26}$.

The phase diagram of the $Q=3$ quantum Potts model is sketched in Fig. 1]

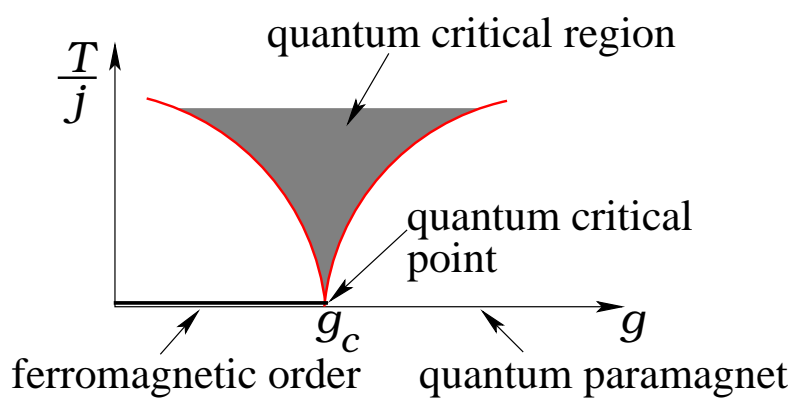

FIG. 1: (Color online) Sketch of the phase diagram of the onedimensional $Q=3$ state quantum Potts model. The quantum fluctuations become dominant at a temperature scale $T \sim \Delta$.

As we indicated in the figure, the quasiparticle gap $\Delta$ approaches zero as a power law at the quantum critical point $g_{c}$,

$$
\Delta_{Q=3}(g) \approx C_{ \pm}\left|g-g_{c}\right|^{5 / 6}
$$

where $C_{ \pm}$denote prefactors corresponding to the $g>g_{c}$ and $g<g_{c}$ regimes. The exponent in Eq. (4) simply follows from the quantum classical mapping between the two-dimensional classical Potts model and the onedimensional quantum Potts model, ${ }^{23,27}$ and is simply the critical exponent $\nu=5 / 6$ of the correlation length $\xi \sim$ $1 / \Delta$ in the two-dimensional classical problem 1.18 .28 .29 In the quantum critical regime, $\Delta<T<j$, physical properties of the model are governed by the zero temperature critical point $g=g_{c}$.

The basic difference between the above two quantum phases can be captured by the change in the dynamical structure factor $S^{\mu \mu^{\prime}}(\omega, q)$, defined as the Fourier transform of the spin-spin correlation function,

$$
S_{\mu, \mu^{\prime}}\left(t, x_{i}\right) \equiv\left\langle\tilde{P}_{i}^{\mu}(t) \tilde{P}_{0}^{\mu^{\prime}}(0)\right\rangle
$$

The structure factor $S^{\mu \mu^{\prime}}(\omega, q)$ is directly measured by neutron scattering in magnetic systems, and it is related to the dynamical susceptibility,

$$
\chi_{\mu, \mu^{\prime}}\left(t, x_{i}\right) \equiv(-i)\left\langle\left[\tilde{P}_{i}^{\mu}(t), \tilde{P}_{0}^{\mu^{\prime}}(0)\right]\right\rangle \theta(t),
$$

through the relation

$$
S_{\mu \mu^{\prime}}(\omega, q)=-2(n(\omega)+1) \operatorname{Im}\left\{\chi_{\mu, \mu^{\prime}}(\omega, q)\right\},
$$

with $n(\omega)$ the Bose function.

In the ferromagnetic phase at $T=0$ temperature the structure factor has a Dirac delta component at $\omega=q=$ 
0

$$
\begin{gathered}
S_{\mu, \mu^{\prime}}^{T=0}(\omega, q)=(2 \pi)^{2} \delta(\omega) \delta(q) m^{2}\left(\delta_{\mu, \tilde{\mu}}-\frac{1}{Q}\right) \\
\left(\delta_{\mu^{\prime}, \tilde{\mu}}-\frac{1}{Q}\right)+\ldots,
\end{gathered}
$$

where the order parameter $m$ is related to the expectation value of $\tilde{P}^{\mu}$ as $\left\langle\tilde{P}^{\mu}\right\rangle=m\left(\delta_{\mu, \tilde{\mu}}-1 / Q\right)$, the order parameter being aligned along direction $\tilde{\mu}$.

The $\omega=q=0$ delta function is a characteristic of long range order and is absent for $g>g_{c}$, where the structure factor has a delta peak at the quasiparticle energy at $T=0$ temperature

$$
S_{\mu, \mu^{\prime}}^{T=0}(\omega, q)=A(g)\left(\delta_{\mu \mu^{\prime}}-1 / Q\right) \delta(\omega-\tilde{\epsilon}(q))+\ldots .
$$

This behavior is schematically shown in Fig. 2 For $Q<$ 4 both the order parameter $m(g)$ and the quasiparticle residue $A(g)$ scale to zero as we approach the quantum critical point $g_{c}$, where the quasiparticle gap $\Delta$ vanishes and the quasiparticle description breaks down.
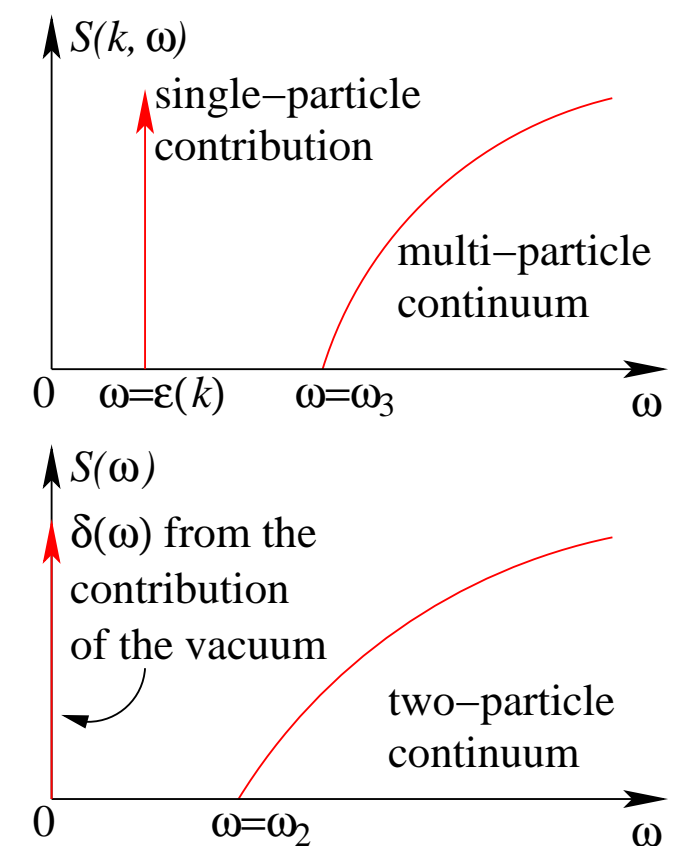

FIG. 2: (Color online) Schematics of the $T=0$ temperature structure factors on the paramagnetic (top) and ferromagnetic (bottom) sides of the phase transition. On the bottom we show the integrated structure factor, $S(\omega) \sim \int d q S(\omega, q)$. Indices are dropped for simplicity.

The $T=0$ temperature dynamics discussed so far gets drastically modified in the gapped phases at finite temperatures where collisions between quasiparticles must also be taken into account. Fortunately, we can get an accurate description of the low temperature dynamics for $T \ll \Delta$ using the semiclassical formalism of Refs. 12 2630].
In this limit, we can describe the spin system as a dilute gas of weakly interacting quasiparticles with momenta $k \approx 0$. Performing a perturbative calculation we find that, in the perturbative regime, for $Q \neq 4$ the scattering matrix $\mathcal{S}$ of these quasiparticles takes on a $\mathrm{SU}(Q-1)$-symmetrical form in agreement with the general arguments of Ref. [26]. In the paramagnetic phase we find, e.g.,

$$
\mathcal{S}_{\lambda, \tilde{\lambda}}^{\lambda^{\prime}, \tilde{\lambda}^{\prime}} \approx(-1) \delta_{\tilde{\lambda}}^{\lambda^{\prime}} \delta_{\lambda}^{\tilde{\lambda}^{\prime}}
$$

thus quasiparticles exchange their internal quantum numbers under the collision while the many-body wave function picks up a phase $(-1)$.

Far on the ferromagnetic side, $g \ll 1$, we also find that the scattering matrix assumes the exchange form above, excepting that now can be viewed as kinks (domain walls) between different vacuum states. In course of the scattering process these kinks exchange their quantum numbers in such a way that the the domain in the "middle" of the collision keeps its color (see Section

Rather surprisingly, for $Q=3$ the results found in the perturbative regime do not agree with the $\mathcal{S}$-matrix that one obtains by removing the cut-off and assuming that the system flows to a massive integrable fixed point ${ }^{31}$ :

$$
\left(\mathcal{S}_{\text {diag }}\right)_{\lambda, \tilde{\lambda}}^{\lambda^{\prime}, \tilde{\lambda}^{\prime}} \sim(-1)^{(\lambda+\tilde{\lambda}) / 2} \delta_{\lambda}^{\lambda^{\prime}} \delta_{\tilde{\lambda}}^{\tilde{\lambda}^{\prime}} .
$$

One, rather unlikely explanation is, that Eq. (10) only describes the scattering of $q \rightarrow 0$ momentum quasiparticles far away from the quantum-critical point, and at for some finite values of $g \neq g_{c}$ 'phase transitions' occur where the asymptotic form of the quasiparticles jumps from one 'universal' form to the other.

However, as we discuss in Appendix [C] rather robust arguments constructed along the lines of Ref. [26] suggest that Eq. (10) is indeed the $\mathcal{S}$-matrix of the $q \rightarrow 0$ momentum quasiparticles on a lattice: The low energy effective field theory contains three independent coupling constants, and unless two of these are the same, the $\mathcal{S}$ matrix takes the form (10) rather than (111).

It is, nevertheless, quite concievable that the previously mentioned two couplings become equal once one removes the cut-off $\Lambda \sim j$.

This in turn means that for $\Delta \ll j$ an intermediate energy regime may exist, where, rather than Eq. (10), the diagonal $\mathcal{S}$-matrix (11) of Ref. 31 describes the scattering processes.

In fact, numerical calculations of the mass spectrum for $Q>3$ seem to support that the above Köberle-Swieca $\mathcal{S}$-matrix correctly describes quasiparticles at large momenta, $q \sim \Delta / c$, where bound solitons are formed ${ }^{32}$

Therefore, while we believe that the correlation functions we derive based upon Eq. (10) always correctly describe the regime $T \rightarrow 0(q \rightarrow 0)$, the range of validity of the results in the regime $\left|g-g_{c}\right| \ll 1$ needs further numerical investigation.

We remark that there are a number of cases where in the 'universal scaling limit' important corrections are 
missed that actually provide the leading contributions to some quantities, so that the Potts model would not be unique at all in this regard:

A famous example is provided by the so-called free fermion point of the sine-Gordon model, where in the continuum limit one also finds a diagonal $\mathcal{S}$-matrix. There too, however, the asymptotic $\mathcal{S}$-matrix assumes the exchange form immediately once one introduces a cut-off or goes slightly away from this special point 33 The same happens in the two-channel Kondo model in the scaling limit. There the channel susceptibility and also that associated with the composite superconducting order parameter simply vanishes in the scaling limit, because the coefficients of these terms are inversely proportional to the cut-of 34 . There are also abundant examples in the literature where the physical properties of a model change dramatically once the integrability condition is violated (random matrix theory, integrable models with just a single impurity, etc.).

The above simple form of the $\mathcal{S}$-matrix, Eq. (10), then enables us to compute analytically the correlation function within the semiclassical approximation . This turns out to be a universal function of the typical separation $\xi_{c}$ between the quasiparticles and their scattering time $\tau$, given explicitely by Eqs. (70) and (71), respectively.

In the ferromagnetic phase we can express the spinspin correlation function for $T \rightarrow 0$ as

$$
S_{\mu, \mu^{\prime}}(x, t)=m^{2} \frac{1}{Q}\left(\delta_{\mu, \mu^{\prime}}-\frac{1}{Q}\right) R(\bar{x}, \bar{t}),
$$

where the relaxation function $R$ is given by the following expression:

$$
\begin{array}{r}
R(\bar{x}, \bar{t})=\int_{-\pi}^{\pi} \frac{d \phi}{2 \pi} e^{-|\bar{t}|(1-\cos \phi)\left(\frac{1}{\sqrt{\pi}} e^{-u^{2}}+u \operatorname{erf}(u)\right)} \\
\cos (\sin (\phi) \bar{x}) \frac{(Q-1)^{2}-1}{(Q-1)^{2}+1+2(Q-1) \cos \phi},
\end{array}
$$

and $\bar{x}=x / \xi_{c}, \bar{t}=t / \tau$, and $u=\bar{x} / \bar{t}$, denote dimensionless separations, times and velocities.

This formula is quite remarkable: On one hand, in the $Q \rightarrow 2$ limit it reproduces the exact results of Refs. [1, 12. However, while for $Q=2$ the function $R$ decays exponentially, for $Q>2$ it has a diffusive structure for $1 \ll \bar{t}$ :

$$
R(\bar{x}, \bar{t}) \sim \frac{1}{\sqrt{\bar{t}}} \exp \left(-\frac{\sqrt{\pi} \bar{x}^{2}}{2 \bar{t}}\right) .
$$

This diffusive structure is related to the approximate $\mathrm{SU}(Q-1)$ invariance of the scattering matrix. As a consequence, the $T=0$ temperature Dirac delta peak in the structure factor is replaced by

$$
S_{\mu \mu^{\prime}}(\omega, q) \sim m^{2}\left(\delta_{\mu, \mu^{\prime}}-\frac{1}{Q}\right) \frac{\tau \xi_{c}^{3} q^{2}}{\xi_{c}^{4} q^{4}+\omega^{2} \tau^{2} 4 \pi}
$$

From this, with the help of (17), we find that the susceptibility in the (semiclassical) limit, $\omega \ll T$ has a diffusion pole,

$$
\chi_{\mu \mu^{\prime}}(\omega, q) \sim m^{2}\left(\delta_{\mu, \mu^{\prime}}-\frac{1}{Q}\right) \frac{\xi_{c}}{T} \frac{\xi_{c}^{2} q^{2}}{\xi_{c}^{2} q^{2}-i \omega \tau 2 \sqrt{\pi}}
$$

The prefactor in this expression, $\sim m^{2} \xi_{c} / T$ is just the static susceptibility, that can be interpreted as the Curie susceptibility of independent domains of size $\xi_{c}$ having magnetization $m$.

In fact, this diffusive structure is rather natural, and we shall discuss a simple explanation for it later. What is not natural, is the exponential decay found in the transverse field Ising model $(Q=2),{ }^{12}$ As we discuss later, this exponential decay is a consequence of long range correlations in the domain wall orientations along the chain for $Q=2$, and is a peculiarity of the Ising model.

For the low temperature correlation function on the paramagnetic side of the phase transition we find the following expression

$$
S_{\mu, \mu^{\prime}}(x, t)=A\left(\delta_{\mu, \mu^{\prime}}-\frac{1}{Q}\right) K(x, t) R(\bar{x}, \bar{t}),
$$

where the propagator $K(x, t)$ is approximately the Feynman propagator of a quantum mechanical particle of mass $\Delta$, and is given by

$$
K(x, t) \approx e^{-i \Delta t} \sqrt{\frac{\Delta}{2 \pi i t}} \exp \left(i \frac{\Delta x^{2}}{2 c^{2} t}\right)
$$

for $x$ and $t$ within the light cone. Remarkably, the relaxation function $R(\bar{x}, \bar{t})$ is the same as the one found in the ferromagnetic phase. This is very likely the consequence of the self-duality of the $Q$-state Potts model 19.22

We have to emphasize that Eqs. (13) and (17) rely on the structure (10) of the $\mathcal{S}$-matrix. Therefore, while they are certainly valid in the regime far from the critical point, $\left|g-g_{c}\right| \sim 1$, it is not clear below what temperature these formulas describe the correlation functions for $\left|g-g_{c}\right| \ll 1$. This issue needs some further numerical investigation which is beyond the scope of the persent paper.

It is important to emphasize that the results Eq. (17) obtained above are also relevant for $S=1$ antiferromagnetic spin chains, $\stackrel{26.35}{\longleftarrow}$ and some two-leg ladder systems that can be mapped to a one-dimensional $O(3)$ quantum rotor model, also equivalent to the $O(3)$ sigma model in the long time and long wavelength limit 1,36 The onedimensional quantum rotor model consists of a chain of ferromagnetically coupled quantum rotors, and is defined as

$$
H_{\text {rotor }}=g \sum_{i} \frac{J}{2} \vec{L}_{i}^{2}-J \sum_{i} \vec{n}_{i} \vec{n}_{i+1}
$$


Here the vectors $\vec{n}_{i}$ denote $N$-dimensional unit vectors and the $\vec{L}_{i}$ 's denote the corresponding angular momentum operators. This model maps to the two-dimensional classical $O(N)$ model. Correspondingly, it does not display a quantum phase transition but has only a paramagnetic phase, where the coupling $g$ always generates a finite gap 1 On the other hand, this paramagnetic phase is similar to that of the $Q$-state Potts model in that the quasiparticles have internal quantum numbers. Let us focus here on the probably most relevant $N=3$ case, the $O(3)$ rotor model, where the gapped quasiparticles are spin $S=1$ objects. Since the scattering matrix takes on the same universal form as in our case ${ }^{26}$, all our calculations of the finite temperature properties carry over to this case as well and give

$$
\langle\vec{n}(x, t) \cdot \vec{n}(0)\rangle=A K(x, t) R_{Q=4}(\bar{x}, \bar{t}),
$$

where $R_{Q=4}$ is just the relaxation function Eq. (13) with $Q=4$.

Thus our results are also relevant to $\operatorname{spin} S=1$ antiferromagnetic chains and also some of the experimentally studied spin ladder systems, 15 for which the one-dimensional $O(3)$ quantum rotor model provides a satisfactory description of low energy (long wavelength) fluctuations 36.37

The semiclassical formalism discussed above gives a consistent description of the dynamical fluctuations at very low temperatures in the gapped phases. Conceptually, however, the most interesting regime is the quantum critical regime, $\Delta<T<j$. In this regime dynamical correlations are governed by fluctuations related to the critical point, $g=g_{c}$, and can be accessed by making use of the conformal invariance of the critical theory. Conformal theory implies that the finite temperature dynamical susceptibility is given approximately by

$$
\begin{aligned}
\chi_{\mu \mu^{\prime}}^{T \gg \Delta}(\omega, q) & \sim\left(\delta_{\mu, \mu^{\prime}}-\frac{1}{Q}\right) \frac{1}{T^{26 / 15}} \\
& \times \frac{\Gamma\left(\frac{1}{15}-i \frac{\omega+c q}{4 \pi T}\right) \Gamma\left(\frac{1}{15}-i \frac{\omega-c q}{4 \pi T}\right)}{\Gamma\left(\frac{14}{15}-i \frac{\omega+c q}{4 \pi T}\right) \Gamma\left(\frac{14}{15}-i \frac{\omega-c q}{4 \pi T}\right)}
\end{aligned}
$$

for $\omega, c q, T>\Delta$, and correspondingly, the susceptibility exhibits $\omega / T$ scaling in this quantum critical regime. A similar scaling form shall be obtained for the local susceptibility.

Although we think they are irrelevant for $T \rightarrow 0$, for completeness, let us also give here the relaxation functions obtained under the assumption of integrability in the gapped phases, i.e. using the diagonal $\mathcal{S}$-matrix, Eq. (11). In this case we obtain the expression

$$
\begin{aligned}
R_{\mathrm{diag}}^{\mathrm{para}}(\bar{x}, \bar{t}) & =e^{-\bar{t} G(\bar{x} / \bar{t})}, \\
G(u) & =\frac{1}{\sqrt{\pi}} e^{-u^{2}}+u \operatorname{erf}(u)
\end{aligned}
$$

for the decay function in the paramagnetic case, while in the ferromagnetic case we find

$$
R_{\text {diag }}^{\text {ferro }}(\bar{x}, \bar{t})=e^{-\frac{3}{2} \bar{t} G(\bar{x} / \bar{t})}
$$

In both cases the correlation function decays exponentially. The derivation of these expressions, which may be relevant in an intermediate temperature range, $\Delta \gg T>T^{*}$ for $\left|g-g_{c}\right| \ll 1$, is given in Appendix [B]

The rest of this paper is organized as follows: First, in Sec. III we investigate shortly the $T=0$ temperature properties of the system in the gapped phases by means of perturbation theory. In Sec. III we use the semiclassical approximation to obtain the low temperature correlation functions. Sec. IV is devoted to the discussion of the quantum critical regime, and our final conclusions are summarized in Sec. [V] Some of the technical details have been relegated to Appendices.

\section{PERTURBATIVE ANALYSIS OF $T=0$ TEMPERATURE PROPERTIES}

In this section we shall study the scattering properties of quasiparticles in the $g \gg 1$ and $g \ll 1$ regimes, where straightforward perturbation theory allows one to obtain the energy of the quasiparticles, understand their structure, and determine their scattering matrix.

\section{A. Paramagnetic side, $g \gg 1$}

We shall first study the limit of very large $g$. In this limit we can perform an expansion in $1 / g$ by taking the term describing the effect of transverse field $\sim g$ as an unperturbed Hamiltonian,

$$
H_{g}=-j g \sum_{i} \tilde{P}_{i}
$$

and considering the ferromagnetic interaction as a perturbation,

$$
H_{\text {ferro }}=-j \sum_{i} \sum_{\mu} \tilde{P}_{i}^{\mu} \tilde{P}_{i+1}^{\mu} .
$$

The ground state of Eq. (25) is simply the paramagnetic state

$$
\left|\lambda_{0}\right\rangle=\prod_{i}\left|\lambda_{0}\right\rangle_{i}
$$

where $\left|\lambda_{0}\right\rangle_{i}$ denotes the state $\left|\lambda_{0}\right\rangle_{i} \leftrightarrow \frac{1}{\sqrt{Q}}(1,1, \ldots, 1)$ at site $i$ in the basis of the ferromagnetic eigenvectors. Elementary excitations in the $g \rightarrow \infty$ limit consist of local 'spin flips' of energy $\delta E=j g$, where one of the spins is in a $\tilde{P}=0$ eigenstate $|\lambda\rangle_{i}$, orthogonal to $\left|\lambda_{0}\right\rangle_{i}$. Obviously, there are $Q-1$ such states, and correspondingly, the label $\lambda$ runs from 1 to $Q-1$. We shall denote the state having Potts spins flipped at sites $i, j, \ldots$ by $\left|i, \lambda_{i} ; j, \lambda_{j} ; \ldots\right\rangle$. 
Due to the ferromagnetic term, Eq. (26), these local excitations can hop between lattice sites and get a dispersion. In leading order in $1 / g$ the corresponding wave function and quasiparticle energy of an elementary excitation is given by

$$
\begin{aligned}
|k ; \lambda\rangle & \approx \sum_{j} e^{i x_{j} k}|j, \lambda\rangle, \\
\epsilon^{\lambda}(k) & \equiv \tilde{\epsilon}(k) \approx j g\left(1-\frac{2}{Q} \frac{1}{g} \cos k\right),
\end{aligned}
$$

where $k$ is the quasi-momentum of the excitations, $x_{j}$ denotes the position of lattice site $j$, and we set the lattice constant to $a=1$.

It is also easy to compute the spin-spin correlation function in this approximation: The operator $\tilde{P}^{\mu}$ creates local spin flip excitations which then propagate along the chain. A simple calculation then gives a single-particle contribution to the dynamical susceptibility

$$
\chi_{\mu \mu^{\prime}}(q, \omega)_{T=0}=\left(\delta_{\mu \mu^{\prime}}-\frac{1}{Q}\right) \frac{A(g) 2 \pi \Delta}{(\omega+i \delta)^{2}-\epsilon_{q}^{2}}+\ldots,
$$

corresponding to a single particle contribution to the structure function given by Eq. (9) with

$$
A(g) \Delta \sim \Delta^{4 / 15} \sim\left(g-g_{c}\right)^{2 / 9}
$$

as one approaches the quantum critical point.

Note that at $T=0$ temperature these quasiparticles have an infinite lifetime to all orders in the perturbation theory (as guaranteed by energy and momentum conservation and the existence of a gap). Of course, Eq. (9) only gives the leading behavior of $S_{\mu \mu^{\prime}}(\omega, q)$, and at higher energies a many-particle continuum also appears above a threshold due to higher order corrections to the ground state wave function.

For $g \gg 1$ the perturbation theory in $1 / g$ is convergent. Therefore the above picture holds to any order in $1 / g$ for $g>g_{c}$, apart from the dispersion $\tilde{\epsilon}(k)$ being renormalized and the quasiparticle weight $A(g)$ being reduced for finite values of $g$. In general the single particle contribution to the correlation function reads,

$$
S_{\mu, \mu^{\prime}}(x, t)=\left(\delta_{\mu, \mu^{\prime}}-\frac{1}{Q}\right) \int \frac{d k}{2 \pi} A_{k} e^{i(k x-\tilde{\epsilon}(k) t)},
$$

with the weight $A_{k}$ related to the matrix elements of $\tilde{P}^{\mu}$ between the exact quasiparticle state $|k ; \lambda\rangle$ and the ground state $|0\rangle$ as $A_{k}=\frac{1}{Q-1} \sum_{\lambda, \mu}\left|\left\langle k ; \lambda\left|\tilde{P}_{x=0}^{\mu}\right| 0\right\rangle\right|^{2}$.

For large values of $x$ and $t$ the integral in Eq. (32) can be evaluated within the saddle point approximation, yielding

$$
S_{T=0}^{\mu, \mu^{\prime}}(x, t)=A(g)\left(\delta_{\mu, \mu^{\prime}}-\frac{1}{Q}\right) K(x, t),
$$

with $K(x, t)$ the Feynman propagator Eq. 18 and $A(g)=\lim _{k \rightarrow 0} A_{k}$.
The spin-spin correlation function further simplifies in the vicinity of the quantum critical point if we assume that the dispersion $\tilde{\epsilon}(k)$ takes on a relativistically invariant form,

$$
\tilde{\epsilon} \approx \sqrt{\Delta^{2}+c^{2} k^{2}},
$$

with $c$ being the quasiparticle velocity, and neglect the $k$-dependence of $A_{k} \rightarrow A(g)$. In this case the zero temperature propagator can be expressed as

$$
K(x, t)=\Delta K_{0}\left(\Delta \sqrt{x^{2}-c^{2} t^{2}} / c\right),
$$

where $K_{0}$ denotes the modified Bessel function. For $Q<$ 4 the quasiparticle gap $\Delta$ and the weight $A$ tend to zero continuously as one approaches the critical values of the coupling, $g_{c}$. For $Q>4$, on the other hand, the phase transition remains first order, and therefore both $A(g)$ and $\Delta$ remain finite at the transition point.

Having analyzed the single particle properties, let us now turn our attention to two-particle states. We look for a two-particle eigenstate of the Hamiltonian by making the following ansatz in leading order in $1 / g$ :

$$
\begin{aligned}
\left|k, k^{\prime}\right\rangle & =\sum_{\lambda, \lambda^{\prime}}\left\{A_{\lambda, \lambda^{\prime}} \sum_{i<j}\left(e^{i\left(k x_{i}+k^{\prime} x_{j}\right)}\left|i, \lambda ; j, \lambda^{\prime}\right\rangle\right)\right. \\
& \left.+B_{\lambda, \lambda^{\prime}} \sum_{i>j}\left(e^{i\left(k x_{i}+k^{\prime} x_{j}\right)}\left|i, \lambda ; j, \lambda^{\prime}\right\rangle\right)\right\} .
\end{aligned}
$$

The coefficients $A_{\lambda, \lambda^{\prime}}$ and $B_{\lambda, \lambda^{\prime}}$ in this ansatz are related by the two-particle $\mathcal{S}$-matrix:

$$
B_{\lambda, \lambda^{\prime}}=\sum_{\tilde{\lambda}, \tilde{\lambda}^{\prime}} \mathcal{S}_{\lambda, \lambda^{\prime}}^{\tilde{\lambda}, \tilde{\lambda}^{\prime}}\left(k, k^{\prime}\right) A_{\tilde{\lambda}, \tilde{\lambda}^{\prime}} .
$$

Substituting (36) into the Schrödinger equation we obtain an equation for $\mathcal{S}_{\lambda, \lambda^{\prime}}^{\tilde{\lambda}, \tilde{\lambda}^{\prime}}\left(k, k^{\prime}\right)$ that can be solved (see Appendix @ for details). The expression of $\mathcal{S}_{\lambda, \lambda^{\prime}}^{\tilde{\lambda}, \tilde{\lambda}^{\prime}}\left(k, k^{\prime}\right)$ is rather involved for general values of $k$ and $k^{\prime}$. However, rather remarkably, for $T \rightarrow 0$, where states with both $k$ and $k^{\prime}$ going to zero are relevant, $\mathcal{S}_{\lambda, \lambda^{\prime}}^{\tilde{\lambda}, \tilde{\lambda}^{\prime}}\left(k, k^{\prime}\right)$ reduces to the simple form already given in the Introduction, Eq. (10):

$$
\mathcal{S}_{\lambda, \tilde{\lambda}}^{\lambda^{\prime}, \tilde{\lambda}^{\prime}} \approx(-1) \delta_{\tilde{\lambda}}^{\lambda^{\prime}} \delta_{\lambda}^{\tilde{\lambda}^{\prime}}
$$

Thus the scattering matrix assumes the very same form as in Ref. [26]. While we obtained this equation in first order perturbation theory, we believe that it is valid to all orders in perturbation theory.

\section{B. Ferromagnetic side, $g \ll 1$}

For $g \ll 1$ we can treat the ferromagnetic part of the Hamiltonian (26) as the unperturbed Hamiltonian, and 
consider the 'transverse field' $g$, Eq. 25. as a perturbation. The ground state of Eq. (26) is $Q$-fold degenerate and corresponds to all spins being aligned in one of the $Q$ possible directions,

$$
|0\rangle_{\mu}=\prod_{j}|\mu\rangle_{j} .
$$

For $g=0$ the excitations of Eq. (26) consist of domain walls,

$$
\left|\mu, \mu^{\prime}\right\rangle_{i}=\prod_{j \leq i}|\mu\rangle_{j} \prod_{i<j^{\prime}}\left|\mu^{\prime}\right\rangle_{j^{\prime}}
$$

and have energy $j$. The local field, Eq. (25), generates a coherent motion of these domain walls. In leading order in $g$ the wave function of the elementary excitations can be written as

$$
|k\rangle_{\mu, \mu^{\prime}} \approx \sum_{j} e^{i k x_{j}}\left|\mu, \mu^{\prime}\right\rangle_{j},
$$

and their energy is obtained by straightforward perturbation theory as

$$
\epsilon_{k}^{\mu, \mu^{\prime}}=j\left(1-g \frac{2}{Q} \cos k+\ldots\right) .
$$

Thus quasiparticles have a gap $\Delta(g) \approx j(1-2 g / Q)$ in this phase too, and the ground state is stable.

In the ground states the expectation value of the operators $\tilde{P}^{\mu}$ is finite,

$$
\left\langle\tilde{\mu}\left|\tilde{P}^{\mu}\right| \tilde{\mu}\right\rangle=\left\{\begin{array}{cl}
m(Q-1) / Q, & \text { if } \mu=\tilde{\mu}, \\
-m / Q, & \text { if } \mu \neq \tilde{\mu} .
\end{array}\right.
$$

Correspondingly, the structure factor has a delta peak associated with the long range order at $\omega=q=0$, given by Eq. (8). We remark here that any finite temperature induces a dilute gas of domain walls which destroy this long range order and broaden the delta peak. As we show in the next section, this delta peak has a Gaussian broadening for $Q=2$, however, becomes a diffusive pole for any $Q>2$.

Similar to the paramagnetic phase discussed in the previous subsection, all the above perturbative results carry over to any finite $g<g_{c}$, because perturbation theory is convergent. For $Q<4$ the order parameter vanishes as one approaches $g_{c}$,

$$
m(g) \sim\left(g_{c}-g\right)^{\beta},
$$

with $\beta$ the order parameter exponent for the twodimensional classical $Q=3$ state Potts model $\left(\beta_{3}=\right.$ $1 / 9)$ and the two-dimensional Ising model $\left(\beta_{2}=1 / 8\right)$, respectively,$\underline{18}$ and the quasiparticle gap vanishes with the same exponent as in the paramagnetic phase, $\nu=5 / 6$ for $Q=3$ and $\nu=1$ for $Q=2$.

We now turn to the study two-particle properties in the limit $g \ll 1$. To keep track of domain wall excitations,

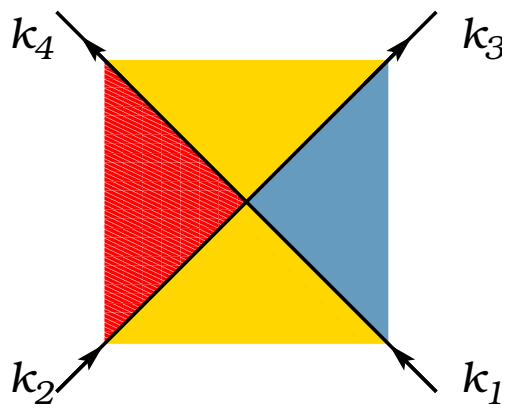

FIG. 3: (Color online) Sketch of colliding domain walls on the ferromagnetic side at $T \ll \Delta$ as $k \rightarrow 0$. Note that the polarization of the middle domain must be conserved and so the order of the domains along the chain does not change with time.

it is worth labelling multi-domain wall configurations in a slightly different way as before. Suppose that we have a domain wall with a polarization $\mu$ on its left and $\mu^{\prime}$ on the right. We can then define the quantum number $\theta=1, \ldots, Q-1$ of this domain wall as the size of the step between the two sides,

$$
\mu^{\prime}=(\mu+\theta) \bmod Q .
$$

Clearly, to characterize any configuration, it is sufficient to give the vacuum state on the left of the chain, and then specify the quantum numbers $\left\{\theta_{j}\right\}$. With this notation, we can thus denote the state described by Eq. (40) as

$$
\left|\mu, \mu^{\prime}\right\rangle_{i} \equiv|i, \theta\rangle_{\mu}
$$

With the above notation, the two-particle wave function has the following form in leading order in $g$,

$$
\begin{aligned}
\left|k, k^{\prime}\right\rangle \equiv & \sum_{\theta, \theta^{\prime}}\left\{A_{\theta, \theta^{\prime}} \sum_{i<j}\left(e^{i\left(k x_{i}+k^{\prime} x_{j}\right)}\left|i, \theta ; j, \theta^{\prime}\right\rangle_{\mu}\right)+\right. \\
& \left.B_{\theta, \theta^{\prime}} \sum_{i>j}\left(e^{i\left(k x_{i}+k^{\prime} x_{j}\right)}\left|i, \theta ; j, \theta^{\prime}\right\rangle_{\mu}\right)\right\}
\end{aligned}
$$

Again, a simple calculation outlined in Appendix $\mathrm{A}$ provides us the scattering matrix $\mathcal{S}_{\tilde{\theta}, \tilde{\theta}^{\prime}}^{\theta, \theta^{\prime}}$ relating the amplitudes $A_{\theta, \theta^{\prime}}$ and $B_{\theta, \theta^{\prime}}$ of the incoming and outgoing particles, respectively. In the limit of vanishing momenta, $k, k^{\prime} \rightarrow 0$ we find

$$
\mathcal{S}_{\tilde{\theta}, \tilde{\theta}^{\prime}}^{\theta, \theta^{\prime}}=(-1) \delta_{\tilde{\theta}}^{\theta^{\prime}} \delta_{\tilde{\theta}^{\prime}}^{\theta},
$$

i.e. quasiparticles scatter as 'hard balls'. This equation can also be visualized as a condition that the orientation of the order parameter between two colliding domain walls with vanishing quasi-momenta remains unchanged after the collision. Again, we believe that the exchange form of the scattering matrix, Eq. [48), remains valid to all orders in $g$ for $g<g_{c}$. 


\section{SEMICLASSICAL APPROXIMATION IN THE GAPPED PHASES}

In this section, we shall study dynamical correlation functions in the $T \ll \Delta$ limit in the gapped phases, following the semiclassical treatment of Refs. 12 26. 30 ]. This approach is based on the observation that at very low temperatures only quasiparticles with energy close to the quasiparticle gap are present, $\epsilon \approx \Delta$. The energy of these quasiparticles can be approximated as

$$
\epsilon_{k}=\Delta+\frac{c^{2}}{\Delta} \frac{k^{2}}{2}+\mathcal{O}\left(k^{4}\right)
$$

with $c$ a constant playing the role of the speed of light, and $\Delta / c^{2}$ the mass of the quasiparticles. For $T \ll \Delta$ the distribution of quasiparticles is described simply by Boltzmann statistics,

$$
n(k) \sim e^{-\beta \Delta} e^{-\beta \frac{c^{2} k^{2}}{2 \Delta}},
$$

with $\beta=1 / T$ the inverse temperature. Correspondingly, the quasiparticle density is exponentially small

$$
\varrho=(Q-1) \sqrt{\frac{T \Delta}{2 \pi c^{2}}} e^{-\Delta / T},
$$

and the typical separation between them, $d_{T} \sim 1 / \varrho$ increases exponentially at low temperatures, $d_{T} \sim e^{\Delta / T}$. This must be compared to the De Broglie wavelength of the particles, $\lambda_{T}$, giving the quantummechanical extension of the quasiparticles' wave function. This latter is given by the inverse of the typical momentum $k$ of the quasiparticles, $\lambda_{T} \sim c / \sqrt{T \Delta}$. Clearly, at very low temperatures the average separation of the quasiparticles is much bigger than their quantum mechanical size,

$$
d_{T} \gg \lambda_{T}
$$

which makes one possible to treat the quantum mechanical state of the system in both phases within the semiclassical approximation for $T \ll \Delta$.

Unfortunately, in one dimension neighboring particles cannot avoid collisions with each other, thus they will get unavoidably closer than the De Broglie wavelength, where quantum mechanics is at work. These collisions must therefore be described within the framework of quantum mechanics. Fortunately, at low $T$, where the semiclassical limit is valid, the system is dilute enough so that we have only 2-particle scattering. Furthermore, the colliding quasiparticles have momenta $k \sim 1 / d_{T}$. Therefore, at low enough temperatures, we can use the simple forms of the scattering matrices given by Eqs. (38) and (48). This simple form of the $\mathcal{S}$-matrix will enable us to obtain analytical results for the correlation functions within the semiclassical picture.

\section{A. Semiclassical correlation function on the ferromagnetic side}

Let us first compute the spin-spin correlation in the less complicated case of ferromagnetic phase. By definition, the time-dependent correlation function is defined as

$$
S_{\mu, \mu^{\prime}}(x, t)=\left\langle e^{i t H} \tilde{P}^{\mu}(x) e^{-i t H} \tilde{P}^{\mu^{\prime}}(0)\right\rangle,
$$

where $\langle\ldots\rangle$ denotes now thermal averaging over all possible many-body states,

$$
\langle\ldots\rangle=\sum_{n}\langle n|\ldots| n\rangle e^{-\beta E_{n}}
$$

where the summation runs over all many-body eigenstates $|n\rangle$ of energy $E_{n}$ of the total Hamiltonian. Within the semiclassical approximation we can replace this average by an average over all possible quasiparticle configurations, i.e., by an average over the quasiparticle velocities $v_{\nu}$, their positions $x_{\nu}$, and internal quantum numbers $\theta_{\nu},(\nu=1, \ldots, M$ with $M$ the number of quasiparticles $)$,

$$
\begin{aligned}
\langle\ldots\rangle \approx & \sum_{\left\{\theta_{\nu}\right\}} \int \prod_{\nu} d x_{\nu} \prod_{\nu} d v_{\nu} P\left(\left\{x_{\nu}, v_{\nu}, \theta_{\nu}\right\}\right) \\
& \left\langle\left\{x_{\nu}, v_{\nu}, \theta_{\nu}\right\}|\ldots|\left\{x_{\nu}, v_{\nu}, \theta_{\nu}\right\}\right\rangle,
\end{aligned}
$$

where the distribution function $P\left(\left\{x_{\nu}, v_{\nu}, \theta_{\nu}\right\}\right)$ is simply

$$
P\left(\left\{x_{\nu}, v_{\nu}, \theta_{\nu}\right\}\right)=\frac{1}{L_{x}^{M}} \frac{1}{(Q-1)^{M}} \prod_{\nu} P\left(v_{\mu}\right)
$$

with $L$ the system size and $P\left(v_{\mu}\right)$ the Boltzmann distribution of the quasiparticle velocities $v=c^{2} k / \Delta$,

$$
P(v)=\sqrt{\frac{\Delta}{2 \pi c^{2} T}} e^{-\frac{\Delta}{c^{2} T} \frac{v^{2}}{2}} .
$$

Of course, in the above equations an average over $M$ should be taken. In the $L \rightarrow \infty$ limit, however, we can replace $M$ by the average particle number, $M \rightarrow \varrho L$, without changing the final result.

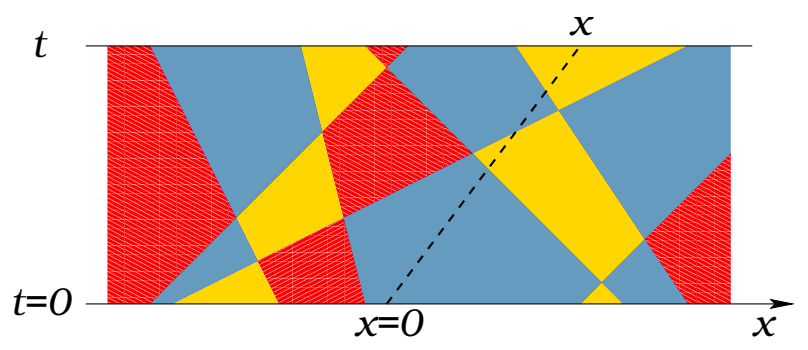

FIG. 4: (Color online) Time evolution of domain walls on the ferromagnetic side at $T \ll \Delta$. For the correlation function $S_{\mu \mu^{\prime}}(x, t)$ we need to take the average over all domain configurations.

To evaluate the correlation function $S_{\mu \mu^{\prime}}(x, t)$, let us first compute the probability $Q_{\mu \mu^{\prime}}(x, t)$, that at time 
$t=0$ and position $x=0$ the order parameter points into direction $\mu$ while at time $t$ and position $x$ it points into direction $\mu^{\prime}$. To evaluate this probability, we notice that the number of domain walls is conserved in course of every domain wall collision. Furthermore, the 'color' (orientation) of the $q$ 'th domain from the left remains unchanged due to the exchange form of the scattering matrix (see Fig. (4). As a consequence, $Q_{\mu \mu^{\prime}}(x, t)$ can be simply written as

$$
\begin{gathered}
Q_{\mu, \mu^{\prime}}(x, t)=\sum_{l, k} P\left((0,0) \text { in } l^{\prime} \text { th }\right) \\
P\left((x, t) \text { in } k^{\prime} \text { th }\right) P\left(l, \mu ; k, \mu^{\prime}\right),
\end{gathered}
$$

i.e. it is simply a product of the probability $P\left((0,0)\right.$ in $l^{\prime}$ th $)$ that $(t=0, x=0)$ is within the $l^{\prime}$ th domain from the left, while the $(t, x)$ point lies in the $k$ 'th domain, and probability that the $l$ 'th domain points in direction $\mu$ while the $k^{\prime}$ th in direction $\mu^{\prime}, P\left(l, \mu ; k, \mu^{\prime}\right)$. We have to remark here that the orientation of domain walls is correlated. This correlation is simply generated by the constraint that if a domain points in a direction $\mu$ then its neighbor must point into one of the other $Q-1$ directions. This correlation is hidden in $P\left(l, \mu ; k, \mu^{\prime}\right)$, which can be computed by constructing a Markov equation, and depends only on $n \equiv l-k, P\left(l, \mu ; k, \mu^{\prime}\right)=P\left(n, \mu, \mu^{\prime}\right)$ :

$$
P\left(n, \mu, \mu^{\prime}\right)=\frac{1}{Q}\left(\frac{-1}{Q-1}\right)^{|n|}\left(\delta_{\mu, \mu^{\prime}}-\frac{1}{Q}\right)+\frac{1}{Q^{2}} .
$$

Note that this correlation function decays exponentially for any $Q>2$, while it oscillates for the Ising model, $Q=2$.

The first part of Eq. (58) also depends only on the separation $n=l-k$ and the coordinates $x$ and $t$. Therefore, Eq. (58) can also be rewritten as

$$
Q_{\mu, \mu^{\prime}}(x, t)=\sum_{n=-\infty}^{\infty} D(n,(x, t)) P\left(n, \mu, \mu^{\prime}\right),
$$

with $P\left(n, \mu, \mu^{\prime}\right)$ given by Eq. (59) and

$$
D(n,(x, t)) \equiv \sum_{l} P\left((0,0) \text { in } l^{\prime} \text { th }\right) P\left((x, t) \text { in } l+n^{\prime} \text { th }\right) .
$$

Clearly, $D(n,(x, t))$ is just the probability that the domain of $(x, t)$ is the $n$ 'th domain to the right from the domain of $(0,0)$.

The probability $D(n,(x, t))$ can be computed as follows. First, following Refs. 26 30], let us introduce the notion of 'particles'. In the configuration space, domain walls are located along straight lines $\nu$,

$$
x_{\nu}(t)=x_{\nu}+v_{\nu} t
$$

where $x_{\nu}$ and $v_{\nu}$ are the position and velocity of the $\nu^{\prime}$ th domain wall at $t=0$. 'Particles', however, correspond to a given step $\theta$ and are 'reflected' when two lines cross.
There is a simple way to tell which particle $p$ is moving along line $\nu$ at time $t$. This is given by the function $p_{\nu}(t)$ which we chose to coincide at $t=0$ with the index $\nu$ of a line, $p_{\nu}(t=0)=\nu$. Particles are, however, impenetrable. Therefore if line $\nu$ is crossed by another line from the left then $p_{\nu}$ decreases by one, while if it is crossed by a line from the right, then it increases by one. Therefore, to keep track of $p_{\nu}(t)$ we just have to count the number of lines that crossed $x_{\nu}(t)$ from the left and from the right since time $t=0$,

$$
p_{\nu}(t)=\nu+\sum_{\nu^{\prime}}\left[\Theta\left(x_{\nu}(t)-x_{\nu^{\prime}}(t)\right)-\Theta\left(x_{\nu}-x_{\nu^{\prime}}\right)\right],
$$

where $\Theta$ denotes the step function.

Similarly, for a fixed set of lines, $\left\{x_{\nu}, v_{\nu}\right\}$, the probability that the straight line from $(0,0)$ to $(x, t)$ moves across $n$ domains to the right is simply

$$
P\left(n \mid\left\{x_{\nu}, v_{\nu}\right\},(x, t)\right)=\delta_{n, \sum_{\nu}}\left[\Theta\left(x-x_{\nu}(t)\right)-\Theta\left(-x_{\nu}\right)\right],
$$

To obtain $D(n,(x, t))$, we should average this expression over $\left\{x_{\nu}, v_{\nu}\right\}$. To do this we just introduce the following integral representation of the Kronecker-delta,

$$
\begin{aligned}
& \delta_{n, \sum_{\nu}\left[\Theta\left(x-x_{\nu}(t)\right)-\Theta\left(-x_{\nu}\right)\right]}= \\
& \int_{-\pi}^{\pi} \frac{d \phi}{2 \pi} e^{i \phi\left(n-\sum_{\nu}\left[\Theta\left(x-x_{\nu}(t)\right)-\Theta\left(-x_{\nu}\right)\right]\right) .}
\end{aligned}
$$

The advantage of this form is that the averaging factorizes and can be evaluated analytically. With some algebra we obtain that

$$
\begin{aligned}
& D(n,(x, t))=\int_{-\pi}^{\pi} \frac{d \phi}{2 \pi} e^{i n \phi} I(x, t)^{M}, \\
& I(x, t)=\left\langle e^{-i \phi\left\{\Theta\left(x-x_{\nu}-v_{\nu} t\right)-\Theta\left(-x_{\nu}\right)\right\}}\right\rangle_{\nu_{\nu}, x_{\nu}} .
\end{aligned}
$$

The integral $I(x, t)$ can then be simply evaluated to yield

$I(x, t)=1-\frac{\bar{t}}{\overline{L_{x}}}\left\{G(u)+G(-u)-e^{i \phi} G(u)-e^{-i \phi} G(-u)\right\}$,

where

$$
G(u)=\frac{1}{2 \sqrt{\pi}} e^{-u^{2}}-\frac{u}{2} \operatorname{erfc}(u) ;
$$

and we introduced dimensionless time and length, $\bar{x}=$ $x / \xi_{c}$, and $\bar{t}=t / \tau$ and the corresponding dimensionless velocity $u=\bar{x} / \bar{t}$, with $\xi_{c}$ and $\tau$ the characteristic classical correlation length and time

$$
\begin{aligned}
\xi_{c} & \equiv 1 / \varrho=\frac{1}{Q-1} \sqrt{\frac{2 \pi c^{2}}{T \Delta}} e^{\Delta / T}, \\
\tau & \equiv \frac{1}{Q-1} \frac{\sqrt{\pi}}{T} e^{\Delta / T} .
\end{aligned}
$$

Finally, $I(x, t)^{M}$ in Eq. can be re-exponentiated as

$$
I(x, t)^{M}=e^{-\bar{t}\left\{G(u)+G(-u)-e^{i \phi} G(u)-e^{-i \phi} G(-u)\right\} .}
$$


Substituting Eqs. (66) and (59) into Eq. (60) the sum over $n$ can be computed using the identity

$$
\sum_{n=-\infty}^{\infty} e^{i \phi n}\left(\frac{-1}{Q-1}\right)^{|n|}=\frac{(Q-1)^{2}-1}{(Q-1)^{2}+1+2(Q-1) \cos \phi}
$$

and the final form of the function $Q_{\mu \mu^{\prime}}(x, t)$ reads:

$$
Q_{\mu \mu^{\prime}}(x, t)=\frac{1}{Q^{2}}+R(\bar{x}, \bar{t}) \frac{1}{Q}\left(\delta_{\mu, \mu^{\prime}}-\frac{1}{Q}\right),
$$

with the relaxation function given by Eq. (13). The correlation function $S_{\mu \mu^{\prime}}(x, t)$ can then be easily computed in terms of $Q_{\mu \mu^{\prime}}(x, t)$ using Eq. (43), as

$$
S_{\mu \mu^{\prime}}(x, t)=\sum_{\tilde{\mu}, \tilde{\mu}^{\prime}}\left\langle\tilde{P}^{\mu}\right\rangle_{\tilde{\mu}}\left\langle\tilde{P}^{\mu^{\prime}}\right\rangle_{\tilde{\mu}^{\prime}} Q_{\tilde{\mu}, \tilde{\mu}^{\prime}}(x, t) .
$$

Putting all this together we finally obtain

$$
S_{\mu \mu^{\prime}}(x, t)=\frac{m^{2}}{Q}\left(\delta_{\mu, \mu^{\prime}}-\frac{1}{Q}\right) R(\bar{x}, \bar{t}) .
$$

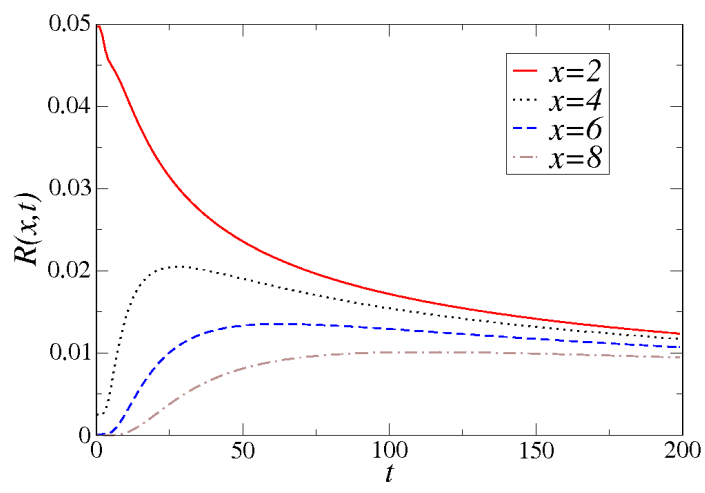

FIG. 5: (Color online) The relaxation function $R(\bar{x}, \bar{t})$ as a function of $\bar{t}$ for different values of $\bar{x}$ at $Q=3$.

The time evolution of $R(\bar{x}, \bar{t})$ is shown in Fig 5 The behavior of the relaxation function $R(\bar{x}, \bar{t})$ is quite interesting for $\bar{t} \gg 1$. The integrand in the definition (13) goes to zero exponentially as $\bar{t} \rightarrow \infty$, and only a small range around $\phi \approx 0$ contributes to the integral. Hence the relaxation function can be approximated as

$R(\bar{x}, \bar{t} \gg 1) \approx \int_{-\infty}^{\infty} \frac{d \phi}{2 \pi Q}(Q-2) e^{-\bar{t} F(u) \phi^{2} / 2} \cos (\phi \bar{x})$,

where $F(u)=1 / \sqrt{\pi} e^{-u^{2}}+u \operatorname{erf}(u)$.

This integral can be calculated analytically and is given by

$$
R(\bar{x}, \bar{t} \gg 1) \approx \frac{Q-2}{Q} \sqrt{\frac{1}{2 \pi \bar{t} F(u)}} e^{-\frac{\bar{x}^{2}}{2 t F(u)}} .
$$

For $\bar{t} \gg \bar{x}$ we have $F(u) \approx 1 / \sqrt{\pi}$, and the relaxation function assumes a diffusive form

$$
R(\bar{x} \ll \bar{t}) \propto \frac{\xi_{c}}{\sqrt{4 \pi t D}} e^{-x^{2} / 4 D t},
$$

with the diffusion constant defined as

$$
D=\frac{1}{2 \sqrt{\pi}} \frac{\xi_{c}^{2}}{\tau}
$$

As a result, the dynamical structure factor has a diffusive structure at $\omega, q \rightarrow 0$, as already discussed in the Introduction.

\section{B. Semiclassical dynamics in the quantum paramagnetic phase}

The analysis of the previous subsection can also be extended to the paramagnetic phase. Similar to the ferromagnetic phase, the average over the many-body eigenstates in Eq. (54) can be replaced in the semiclassical limit by an average over all possible initial states, $\left|\left\{x_{\nu}, v_{\nu}, \lambda_{\nu}\right\}\right\rangle$. The main difference in the calculation is that on the paramagnetic side the operator $\tilde{P}^{\mu}(0)$ in Eq. (53) creates a quasiparticle with some internal quantum number $\lambda_{0}$, and velocity $v$ at time $t=0$ with some probability amplitude $e_{\lambda_{0}}^{\mu}(v)$ in addition to the already existing quasiparticles. This particle, together with the other quasiparticles, propagates under the action of $H$ in Eq. (53) ) and collides with them. For very small temperatures, however, the two-particle scattering matrix takes on an exchange form, and therefore particles only exchange their velocity while conserving their internal quantum numbers.

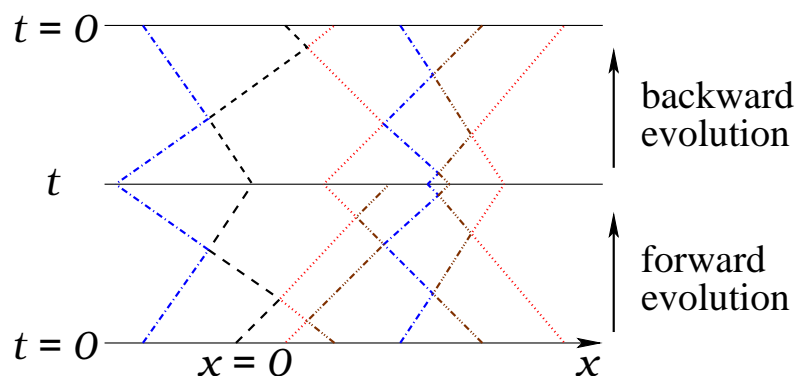

FIG. 6: (Color online) To evaluate the correlation function on the paramagnetic side, one must keep track of the particle created by the operator $\tilde{P}^{\mu}$. Lines drown with different line-styles denote trajectories of different 'particles'. The two states after and before the forward and backward time evolution must be identical. Therefore, the internal quantum numbers of the particles must obey constraints.

As a consequence, at any time precisely one of the particles will have the velocity $v$ of the initial particle and it will be at position $v t$. It is easy to see that this very particle must be annihilated at time $t$ by $\tilde{P}^{\mu^{\prime}}(x)$ otherwise the final state obtained after the action of $e^{i t H}$ in Eq. (53) will be orthogonal to the initial state. (The evolution of these quasiparticles is shown in Fig. 6] The probability amplitude that this particle is annihilated is proportional to $\left(e_{\lambda^{\prime}}^{\mu}(v)\right)^{*} e^{i k x}$ with $k=\Delta v / c^{2}$ the quasi-momentum that corresponds to $v$ and $\lambda$ the internal quantum number of 
the particle that is removed. As we shall see below, $\lambda^{\prime}$ in this expression must be equal to $\lambda_{0}$. This shall be guaranteed by another requirement, namely that the internal quantum numbers in the final state must be exactly the same as those of the initial state. To see what this condition implies let us assume that in the initial state we had quasiparticles with quantum numbers $\left\{\lambda_{1}, \lambda_{2}, \ldots\right\}$ at positions $x_{\mu}\left(x_{1}<x_{2}<\ldots\right)$ with velocities $v_{\mu}$, and that the new particle is created right after the $p$ 'th particle

$$
\begin{aligned}
\tilde{P}^{\mu}(0): & \left\{\ldots, \lambda_{p}, \lambda_{p+1}, \ldots\right\} \\
\longmapsto & \left.\longmapsto \ldots, \lambda_{p}, \lambda_{0}, \lambda_{p+1}, \ldots\right\} .
\end{aligned}
$$

Let us now define the function $p_{0}(t)$ which gives us the label of that particle which has velocity $v$ at time $t$. Obviously, $p_{0}(t=0)=0$. The important observation is that the above order of these quantum numbers from the left to the right along the chain does not change under the collisions due to the simple form of the scattering matrix Eq. (38). Suppose now that after the time evolution $t$ particle ' $n+p$ ' moves with velocity $v$, i.e., $p_{0}(t)$ takes the value $p_{0}(t)=p+n$. (Here we assume for the sake of simplicity that $n>0$, but the derivation can be easily generalized for $n \leq 0$.) Since the operator $\tilde{P}^{\mu^{\prime}}$ must destroy precisely this particle of velocity $v, \tilde{P}^{\mu^{\prime}}$ therefore changes the series of quantum numbers as

$$
\begin{aligned}
& \tilde{P}^{\mu^{\prime}}: \quad\left\{\ldots, \lambda_{p}, \lambda_{0}, \lambda_{p+1}, \ldots\right. \\
& \left.\ldots, \lambda_{p+n-1}, \lambda_{p+n}, \lambda_{p+n+1}, \ldots\right\} \\
& \longmapsto\left\{\ldots, \lambda_{p}, \lambda_{0}, \lambda_{p+1}, \ldots\right. \\
& \left.\ldots, \lambda_{p+n-1}, \lambda_{p+n+1}, \ldots\right\} \text {. }
\end{aligned}
$$

Since the order of these quantum numbers does not change under the action of $e^{-i t H}$ either, requiring that the internal quantum numbers of the initial states be the same as those of the final state amounts in the condition

$$
\lambda_{0} \equiv \lambda_{p+1} \equiv \ldots \equiv \lambda_{p+n}
$$

Now, similar to the ferromagnetic case, the value of $n$ in the previous expressions can be simply computed as the number $N_{+}$of lines $x_{\nu}(t)$ that cross the line connecting $(0,0)$ with $(x, t)$ from the right minus the number of lines $N_{-}$that cross it from the left,

$$
n=\sum_{\nu}\left[\Theta\left(x(t)-x_{\nu}(t)\right)-\Theta\left(0-x_{\nu}\right)\right]
$$

where $\nu$ labels the quasiparticles in the initial state, $x_{\nu}(t)=x_{\nu}+v_{\nu} t$, and $x(t)=v t$ is the trajectory of the new particle with velocity $v$ is created by $\tilde{P}^{\mu}(0)$ at the origin, $x(0)=0$.

To compute the contribution of this particular state to the correlation function we must consider the important detail of phase factors. Quasiparticles in the initial state generate a phase factor $e^{-i t \sum_{\nu} \epsilon\left(v_{\nu}\right)}$ under the action of $e^{-i t H}$. This is, however, completely canceled under the action of $e^{i t H}$, except for the quasiparticle created by $\tilde{P}^{\mu}$ giving a factor $e^{-i t \epsilon(v)}$. Furthermore, every collision results in a sign change of the many-body wave function. All these signs cancel under the forward and backward propagation, excepting the ones that are associated with collisions with the extra particle. These give an extra sign $(-1)^{N_{+}+N_{-}}$, which can, however be also written more conveniently as

$$
S=(-1)^{N_{+}-N_{-}}=(-1)^{n}
$$

Putting all these together we obtain the following expression for the correlation function,

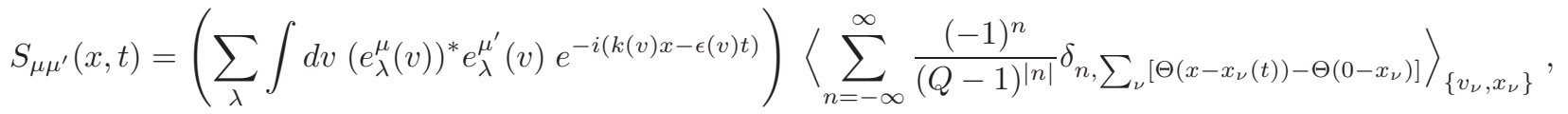

where the factor $1 /(Q-1)^{|n|}$ comes from the condition Eq. (83) after averaging over all possible internal quantum numbers. The first term in this expression gives just the $T=0$ correlation function Eq. (33), while the second part is the same relaxation function as the one found in the ferromagnetic phase, Eq. (13),

$$
S_{\mu, \mu^{\prime}}(x, t)=S_{T=0}^{\mu, \mu^{\prime}}(x, t) R(\bar{x}, \bar{t}) .
$$

Finally, let us briefly discuss the properties of quasiparticle density-density correlations in the paramagnetic phase. The density of quasiparticles with quantum num- ber $\lambda$ is simply given by

$$
\varrho_{\lambda}(x, t)=\sum_{\nu} \delta_{\lambda, \lambda_{p_{\nu}(t)}} \delta\left(x_{\nu}(t)-x\right)
$$

where the summation runs over all lines. The average density, $\varrho \equiv\langle\varrho(x, t)\rangle$ is simply given by Eq. (51). The density-density correlation function can be computed analytically within the semiclassical approximation. We shall not discuss this calculation here, since it is basically identical to the computation of Damle and Sachdev 26 The result is simply 
$\left\langle\varrho_{\lambda}(x, t) \varrho_{\lambda^{\prime}}(0)\right\rangle=\varrho^{2} \frac{\delta_{\lambda, \lambda^{\prime}}}{Q-1} e^{-\bar{t}\left(G_{+}+G_{-}\right)} \times\left\{\left(\frac{e^{-u^{2}}}{\sqrt{\pi} \bar{t}}+2 C_{+} C_{-}\right) I_{0}\left(2 \bar{t} \sqrt{G_{+} G_{-}}\right)+\frac{C_{+}^{2} G_{-}+C_{-}^{2} G_{+}}{\sqrt{G_{+} G_{-}}} I_{1}\left(2 \bar{t} \sqrt{G_{+} G_{-}}\right)\right\}$

where $G_{ \pm}$and $C_{ \pm}$denote the functions

$$
\begin{aligned}
G_{ \pm} & \equiv G( \pm u)=\frac{1}{2 \sqrt{\pi}} e^{-u^{2}} \mp \frac{u}{2} \operatorname{erfc}( \pm u), \\
C_{ \pm} \equiv C( \pm u) & =\frac{1}{2} \operatorname{erfc}( \pm u),
\end{aligned}
$$

with $u=\bar{x} / \bar{t}$ the dimensionless velocity defined earlier.

For large time scales, $\bar{t} \gg 1$ the density-density correlation function also displays a diffusive behavior $\stackrel{26}{\underline{26}}$

$$
\left\langle\varrho_{\lambda}(x, t) \varrho_{\lambda^{\prime}}(0)\right\rangle \sim \delta_{\lambda, \lambda^{\prime}} \frac{1}{\sqrt{\bar{t}}} e^{-\sqrt{\pi} \bar{x}^{2} / 2 \bar{t}^{2}},
$$

in agreement with the behavior of the relaxation function $R(\bar{x}, \bar{t})$. In the $Z_{3}$ case, this correlation function also bears physical meaning and corresponds to the correlation function of the chirality density.

\section{DYNAMICS IN THE QUANTUM CRITICAL REGION}

In this section we shall focus to the $Q=3$ Potts model which has a quantum critical point at $T=0$ and $g=g_{c}$. We can gain many information already from the quantum-classical mapping, by just using the scaling properties of the singular part of the free energy density:

$$
f\left(g-g_{c}, h, T\right)=b^{-2} f\left(b^{y_{t}}\left(g-g_{c}\right), b^{y_{h}} h, T b\right),
$$

where $y_{t}=2-x_{t}$ and $y_{h}=2-x_{h}$ denote the scaling dimensions of the temperature and the magnetic field in the classical Potts model, and $x_{t}=4 / 5$ and $x_{h}=2 / 15$ denote the dimensions of the corresponding primary fields that are known from conformal field theory ${ }^{18.21}$ Note that the temperature plays the role of a finite system size while the coupling $g$ corresponds to the temperature.

From Eq. (931) immediately follows that the gap vanishes as $\Delta \sim\left|g-g_{c}\right|^{5 / 6}$, and that the susceptibility behaves in the quantum critical region as

$$
\chi(T>\Delta) \sim \frac{1}{T^{26 / 15}} .
$$

For $T<\Delta$ the susceptibility becomes finite, but it diverges as one approaches the quantum critical point,

$$
\chi(T=0) \sim \frac{1}{\left|g-g_{c}\right|^{13 / 9}} .
$$

So far, we only discussed thermodynamical properties. However, a lot of information can also obtained by making use of the conformal invariance of the critical theory.
At the critical point, the imaginary time correlation function is scale invariant and has the following form:

$$
\begin{aligned}
\chi_{\mu \mu^{\prime}}^{T=0}(x, \tau) & =\left(\delta_{\mu \mu^{\prime}}-\frac{1}{Q}\right) \chi(x, \tau), \\
\chi(x, \tau) & \sim \frac{1}{\left(\tau^{2}+x^{2}\right)^{x_{h}}} .
\end{aligned}
$$

For simplicity, we set the 'speed of light' to one in this section.

To obtain the finite temperature imaginary time susceptibility (retarded correlation function), we introduce new, complex coordinates $z \equiv \tau+i x$ and $\bar{z}=\tau-i x$ and rewrite (97) as

$$
\chi^{T=0}(z, \bar{z}) \sim \frac{1}{z^{x_{h}} \bar{z}^{x_{h}}} .
$$

Then we map the complex plane to a strip of width $\beta=$ $1 / T$ by the transformation

$$
w=\frac{1}{\pi T} \operatorname{cotan}(\pi T z),
$$

and use the transformation properties of primary fields to obtain $\underline{18}$

$$
\chi(x, \tau) \sim \frac{T^{2 x_{h}}}{(\sin (\pi T(\tau-i x)))^{x_{h}}(\sin (\pi T(\tau+i x)))^{x_{h}}} .
$$

To obtain the (retarded) susceptibility $\chi(\omega, q)$ one has to Fourier transform this correlation function to obtain the Matsubara Green's function, $\chi\left(\omega_{n}, q\right)$ and then analytically continue it back to the real axis $\frac{1.5}{1.5}$ The susceptibility has the same structure as the one obtained for the transverse field Ising model,$\frac{1}{,}$

$$
\chi(T, \omega, q) \sim \frac{1}{T^{26 / 15}} \frac{\Gamma\left(\frac{1}{15}-i \frac{\omega+q}{4 \pi T}\right) \Gamma\left(\frac{1}{15}-i \frac{\omega-q}{4 \pi T}\right)}{\Gamma\left(\frac{14}{15}-i \frac{\omega+q}{4 \pi T}\right) \Gamma\left(\frac{14}{15}-i \frac{\omega-q}{4 \pi T}\right)} .
$$

This immediately implies that the dynamical susceptibility $\chi(\omega) \equiv \chi(\omega, q=0)$ shows $\omega / T$ scaling,

$$
\chi(\omega, T)=\frac{C}{T^{26 / 15}} F(\omega / T),
$$

where the scaling function $F(y)$ is given by

$$
F(y)=\left(\frac{\Gamma\left(\frac{14}{15}\right)}{\Gamma\left(\frac{1}{15}\right)} \frac{\Gamma\left(\frac{1}{15}-i \frac{y}{4 \pi}\right)}{\Gamma\left(\frac{14}{15}-i \frac{y}{4 \pi}\right)}\right)^{2}
$$

and has the following asymptotic properties:

$$
F(y) \approx \begin{cases}1 & y \ll 1 \\ {\left[\frac{\Gamma(14 / 15)}{\Gamma(1 / 15)}\right]^{2}(y / 4 \pi)^{-26 / 15} e^{i \pi 13 / 15}} & y \gg 1\end{cases}
$$


Another quantity of interest is the local susceptibility, $\chi^{\mathrm{loc}}(\omega) \equiv \chi(\omega, x=0)$, that can also be measured by neutron scattering, and which can be computed to behave as

$$
\chi^{\text {local }}(\omega, T)=\frac{\tilde{C}}{T^{11 / 15}} G(\omega / T)
$$

with the scaling function

$$
G(y)=\frac{\Gamma\left(\frac{13}{15}\right)}{\Gamma\left(\frac{2}{15}\right)} \frac{\Gamma\left(\frac{2}{15}-i \frac{y}{2 \pi}\right)}{\Gamma\left(\frac{13}{15}-i \frac{y}{2 \pi}\right)},
$$

having the asymptotic properties:

$$
G(y) \approx \begin{cases}1 & y \ll 1 \\ \frac{\Gamma(13 / 15)}{\Gamma(2 / 15)}(y / 2 \pi)^{-11 / 15} e^{i \pi 11 / 30} & y \gg 1\end{cases}
$$

We have to emphasize that, although conformal invariance only holds at the quantum critical point, the above expressions also apply to the entire quantum critical region, $j \gg T, \omega, q \gg \Delta$, where the quasiparticle gap does not play an important role.

\section{CONCLUSIONS}

In this paper we studied dynamical properties in the gapped phases of the $Q$-state Potts model in the $T \rightarrow 0$ limit, and in its quantum critical behavior for $Q=3$ (the $Q=2$ Ising case has already been studied thoroughly in the literature). Deep in the gapped phases the $T \rightarrow 0$ correlation functions were found to show a diffusive character for $Q>2$. This is a consequence of the simple structure of the low energy scattering matrices, , and can be understood in a very simple way. Consider, for the sake of simplicity, the ferromagnetic side, $T \ll \Delta, g<g_{c}$. In this limit domain walls propagate as particles and eventually collide with other domain walls. However, due to the simple structure of the scattering matrix the order of the color of domains does not change under these collisions.

Therefore, if we look at a given domain, then its size and position changes in time, but its color (orientation) does not. In other words, each domain has a typical size $\sim \xi_{c}$ and a given domain diffuses along the chain as time flows. The colors of domains far away from each other are uncorrelated. Therefore, the probability that the domain at time $t$ at position $x$ has the same color as the domain at $t=0$ and $x=0$ is approximately given by the probability that the domain wall that was at position $x=0$ at time $t=0$ diffused to position $x$ under time $t$, and is therefore proportional to

$$
S_{\mu \mu^{\prime}} \sim \frac{1}{\sqrt{4 \pi t D}} e^{-x^{2} / 4 D t},
$$

with $D$ the diffusion constant of domains, given analytically by Eq. (80).
This simple argument, however, obviously fails for $Q=2$, where the colors of domains far away from eachother are strongly correlated, as illustrated in Fig. 7 Mathematically, from Eq. (59) we see that correlations between the colors of two domains separated by $n$ domain walls decay exponentially for $Q>2, C \sim e^{-n \ln (Q-1)}$, while they simply oscillate for $Q=2$. These correlations for $Q=2$ lead to a destructive 'interference' and give rise to an exponential decay of the correlation functions for the Ising case, $Q=2.12$ Note that the $Q=2$ Potts model is integrable ${ }^{38}$, and a similar exponential decay has also been found recently by Altshuler and Tsvelik in some other exactly integrable cases 39

On the other hand, after this work was completed, we became aware of independent work by Damle and Sachdev $\stackrel{40}{\longleftarrow}$ who find that diffusive behavior appears in the correlators of the one-dimensional sine-Gordon model too. Rather remarkably, the decay functions in the two cases seem to differ only in the choice of the parameter $Q-1$, which takes a value $Q-1 \rightarrow-1 / \cos (2 \pi \eta / \gamma)$ in the sine-Gordon calculation, although the two models are completely different. This similarity between the two results indicates that the form of correlation function we have found is remarkably universal.

We have to emphasize that the diffusive character of the correlation functions for $Q>2$ is a consequence of the simple structure (10) of the scattering matrix between quasiparticles of vanishingly small momenta. At finite temperature, however, the colliding particles have finite momenta $\sim \sqrt{T \Delta / c^{2}}$, and therefore the quantum numbers of the colliding particles change with a finite probability in course of a collision. As a consequence, the color of the domain between the two colliding domain walls can change with a finite probability. At long enough time scales, this process should lead to a decay of correlations, unless the correlation function of the density of a conserved quantity is computed 26

The probability of color changing can be estimated to be proportional to $\sim\left\langle a^{2}(\Delta p)^{2}\right\rangle$ with $\Delta p$ the typical momentum difference of the two incoming particles and $a$ the lattice constant, and scales as $P_{\text {flip }} \sim a^{2} \Delta T / c^{2}$. A given domain shrinks to zero due to the motion of domain walls approximately $t / \tau=\bar{t}$ times during a time period $t$, and each time it shrinks to zero it changes color with the above probability.

As a result, the diffusive form of the decay function in the quantum Potts model must break down at a time scale

$$
t_{\mathrm{diff}} \sim \tau \frac{c^{2}}{a^{2} \Delta T}
$$

above which correlation functions must decay exponentially.

We also present very robust renormalization group arguments to show that on the lattice the asymptotic $\mathcal{S}$ matrix generically takes the exchange form found in the perturbative regimes, and thus the diffusive correlation function we found should also describe the vicinity of 
Ising model

\begin{tabular}{|c|c|c|c|c|c|c|}
\hline- & + & - & + & - & + & - \\
\hline
\end{tabular}

\section{Potts model}

\begin{tabular}{lllllllll}
1 & 2 & 3 & 1 & 2 & 1 & 3 \\
\hline
\end{tabular}

FIG. 7: Domain structure in the transverse field Ising model and in the $Q=3$ state quantum Potts model. For $Q=2$ orientations of far away domains are correlated while for $Q>$ 2 they are not.

the quantum critical point as $T \rightarrow 0,\left|g-g_{c}\right| \ll 1$. In this regime, however, further numerical calculations are needed to determine the applicability and range of validity of our formulas.

Our results are not valid for the case $Q=4$, where scattering matrix cannot be computed in the way outlined in Appendix II] because the matrices become singular for $k, k^{\prime} \rightarrow 0$, and cannot be inverted. As a consequence, the scattering matrix has a singular structure for $k, k^{\prime} \rightarrow 0$, and a special treatment is needed. Indeed, the $Q=4$ state Potts model is known to have unusual thermodynamical properties due to the presence of a marginal operator at the critical point 25

Also, in the present paper we neglected umklapp processes. It has been shown recently, that in one dimension such processes may dominate the relaxation of some currents that overlap with other conserved quantities. 41

These processes are, however, probably not relevant for the correlation functions computed here, since "spin conservation" in course of the scattering process is anyway only approximate in the Potts model. These umklapp processes are, however, probably important for energy relaxation, which has not been studied in this paper.

Finally, let us discuss physical applications of our calculations. Though the 3-state quantum Potts model can be realized in trigonal ferromagnets in a magnetic field, and also recently some spin chain models have been shown to map on this model, 42 it is physically not easy to find realizations of the 1-dimensional $Q$-state quantum Potts model. However, our results obtained for the paramagnetic phase are very general, and carry over to gapped antiferromagnets and spin ladders with little modifications. For the spin-spin correlation function of a one $S=1$ spin Heisenberg antiferromagnet, e.g., our results imply that the spin-spin correlation function decays as

$$
\left\langle\vec{S}\left(x_{i}, t\right) \cdot \vec{S}(0)\right\rangle=(-1)^{i} A K\left(x_{i}, t\right) R_{Q=4}\left(\bar{x}_{i}, \bar{t}\right),
$$

with the Feynman propagator $K$ and the decay function $R$ given by equations (18) and (13). This result implies that the dynamical susceptibility has an approximate diffusive structure also at momentum $q=\pi / a$, similar to the diffusive structure that appears at $q=0.26$ However, while the $q=0$ diffusion pole follows from the SU(2)invariance of the Hamiltonian and is therefore protected by symmetry, the $q=\pi / a$ diffusive character is only approximate, and is a consequence of the simple exchange form of the scattering matrix.

We would like to express our special thanks to Kedar Damle and Subir Sachdev for showing us their preprint before making it public, and F. Iglói for valuable comments and discussions. We would also like to thank A. Tsvelik and R. Konik for useful discussions. This research has been supported by Hungarian Grants No. OTKA T046303, T046267, and NF061726.

\section{APPENDIX A: DERIVATION OF THE SCATTERING MATRICES}

\section{Scattering matrices - ferromagnetic side}

To obtain the two-particle scattering matrix in leading order in $g$ we make the simple ansatz Eq. (47) for the two-particle wave function $|\Psi\rangle$,

$$
\begin{aligned}
|\Psi\rangle= & \sum_{\theta, \theta^{\prime}}\left\{A_{\theta, \theta^{\prime}} \sum_{i<j}\left(e^{i\left(k x_{i}+k^{\prime} x_{j}\right)}\left|i, \theta ; j, \theta^{\prime}\right\rangle_{\tilde{\mu}}\right)+\right. \\
& \left.B_{\theta, \theta^{\prime}} \sum_{i>j}\left(e^{i\left(k x_{i}+k^{\prime} x_{j}\right)}\left|i, \theta ; j, \theta^{\prime}\right\rangle_{\tilde{\mu}}\right)\right\},
\end{aligned}
$$

where $\tilde{\mu}$ is the orientation of the chain on the far left, and $\theta$ and $\theta^{\prime}$ denote the two kinks corresponding to the two domain walls. In leading order in $g$ and for coordinates $|i-j| \gg 1, H_{g}$ just moves the two domain walls independently, and $|\Psi\rangle$ is clearly an eigenstate of the total Hamiltonian with an eigenvalue

$$
H|\Psi\rangle=\left(E_{0}+\epsilon(k)+\epsilon\left(k^{\prime}\right)\right)|\Psi\rangle
$$

$E_{0}$ being the ground state energy, and $\epsilon(k)$ the quasiparticle energy given by Eq. (42). However, $|\Psi\rangle$ must satisfy Eq. A2 also for $j=i+1$, i.e. for nearest neighbors. Observing that the operator $H_{g}=-j g \sum_{n} \tilde{P}_{n}$ just flips each spin to some other direction, we can write in the original notation

$$
\begin{aligned}
& \sum_{n} \tilde{P}_{n}\left|\tilde{\mu}, i, \mu, i+1, \mu^{\prime}\right\rangle= \\
& \langle\tilde{\mu}|\tilde{P}| \mu\rangle\left|\tilde{\mu}, i-1, \mu, i+1, \mu^{\prime}\right\rangle+ \\
& \left\langle\mu|\tilde{P}| \mu^{\prime}\right\rangle\left|\tilde{\mu}, i, \mu, i+2, \mu^{\prime}\right\rangle+ \\
& \quad \sum_{\mu^{\prime \prime} \neq \mu}\left\langle\mu^{\prime \prime}|\tilde{P}| \mu\right\rangle\left|\tilde{\mu}, i, \mu^{\prime \prime}, i+1, \mu^{\prime}\right\rangle+\ldots,
\end{aligned}
$$

where $\theta=(\mu-\tilde{\mu}) \bmod Q, \theta^{\prime}=\left(\mu^{\prime}-\mu\right) \bmod Q$, and we neglected all other terms involving more than two domain wall excitations, since these are high up in energy. Since the off-diagonal matrix elements of $\tilde{P}_{n}$ are all equal to 
$1 / Q$, we can write this in the 'kink' representation as:

$$
\begin{aligned}
& \sum_{n} \tilde{P}_{n}\left|i, \theta ; i+1, \theta^{\prime}\right\rangle_{\tilde{\mu}}= \\
& \frac{1}{Q}\left[\left|i-1, \theta ; i+1, \theta^{\prime}\right\rangle_{\tilde{\mu}}+\left|i, \theta ; i+2, \theta^{\prime}\right\rangle_{\tilde{\mu}}+\right. \\
& \left.\sum_{\tilde{\theta}, \tilde{\theta}^{\prime}} \hat{\delta}_{\theta+\tilde{\theta}^{\prime}}^{\tilde{\theta}^{\prime}+\tilde{\theta}^{\prime}}\left(1-\delta_{\theta}^{\tilde{\theta}^{\prime}}\right)\left|i, \tilde{\theta}^{\prime} ; i+1, \tilde{\theta}\right\rangle_{\tilde{\mu}}\right]+\ldots
\end{aligned}
$$

where $\hat{\delta}$ denotes the Kronecker delta modulo $Q$. Projecting out the $i+1=j$ component of the Schrödinger equation Eq. A2 we obtain the following constraint for the coefficients $A_{\theta, \theta^{\prime}}$ and $B_{\theta, \theta^{\prime}}$,

$$
\begin{aligned}
& \sum_{\tilde{\theta}, \tilde{\theta}^{\prime}}\left[\delta_{\theta}^{\tilde{\theta}} \delta_{\theta^{\prime}}^{\tilde{\theta}^{\prime}}+\frac{1}{e^{i k^{\prime}}+e^{-i k}} \hat{\delta}_{\theta+\theta^{\prime}}^{\tilde{\theta}+\tilde{\theta}^{\prime}}\left(1-\delta_{\theta}^{\tilde{\theta}^{\prime}}\right)\right] B_{\tilde{\theta}^{\prime}, \tilde{\theta}}= \\
& -\sum_{\tilde{\theta}, \tilde{\theta}^{\prime}}\left[\delta_{\theta}^{\tilde{\theta}} \delta_{\theta^{\prime}}^{\tilde{\theta}^{\prime}}+\frac{1}{e^{i k}+e^{-i k^{\prime}}} \hat{\delta}_{\theta+\theta^{\prime}}^{\tilde{\theta}+\tilde{\theta}^{\prime}}\left(1-\delta_{\theta}^{\tilde{\theta}^{\prime}}\right)\right] A_{\tilde{\theta}, \tilde{\theta}^{\prime}} .
\end{aligned}
$$

This equation can clearly be inverted to give the twoparticle $\mathcal{S}$-matrix in leading order in $g$, but the solution is rather complicated even for $Q=3$. Eq. A5 simplifies, however, in the limit $k, k^{\prime} \rightarrow 0$, relevant for very small temperatures, $T \ll \Delta$.

For $Q=3$ we obtain in this way

$$
\mathcal{S}_{\theta, \theta^{\prime}}^{\tilde{\theta}, \tilde{\theta}^{\prime}}\left(k, k^{\prime} \rightarrow 0\right)=(-1) \delta_{\theta}^{\tilde{\theta}^{\prime}} \delta_{\theta^{\prime}}^{\tilde{\theta}} .
$$

We remark here that the above result holds for any $Q \neq 4$. The $Q=4$ case, however, seems to be special: then the operator in front of the coefficients $A_{\tilde{\theta}, \tilde{\theta}^{\prime}}$ and $B_{\theta, \theta^{\prime}}$ in Eq. A5 has zero eigenvalues for $k=k^{\prime}=0$, the inversion is problematic, and the $\mathcal{S}$-matrix does not take the form $\mathrm{A} 5$.

\section{Scattering matrices - paramagnetic side}

To obtain the two-particle scattering matrix in leading order in $1 / g$ we follow similar steps as for the ferromagnetic case. The ansatz for the two-particle wave function can be written as follows:

$$
\begin{aligned}
|\tilde{\Psi}\rangle= & \sum_{\lambda, \lambda^{\prime}}\left\{A_{\lambda, \lambda^{\prime}} \sum_{i<j}\left(e^{i\left(k x_{i}+k^{\prime} x_{j}\right)}\left|i, \lambda ; j, \lambda^{\prime}\right\rangle\right)+\right. \\
& \left.B_{\lambda, \lambda^{\prime}} \sum_{i>j}\left(e^{i\left(k x_{i}+k^{\prime} x_{j}\right)}\left|i, \lambda ; j, \lambda^{\prime}\right\rangle\right)\right\} .
\end{aligned}
$$

For $|i-j| \gg 1|\tilde{\Psi}\rangle$ must satisfy a similar two-particle Schrödinger equation as A2 and so it must be valid for $j=i+1$ too. Let us now calculate the effect of $H_{\text {ferro }}=-j \sum_{n} \sum_{\mu} \tilde{P}_{n}^{\mu} \tilde{P}_{n+1}^{\mu}$ on the $i+1=j$ term of $|\tilde{\Psi}\rangle$. If we neglect the high energy terms with multiple particles we get

$$
\sum_{n} \sum_{\mu} \tilde{P}_{n}^{\mu} \tilde{P}_{n+1}^{\mu}\left|i, \lambda ; i+1, \lambda^{\prime}\right\rangle=
$$

$$
\begin{array}{r}
1 / Q\left|i-1, \lambda ; i+1, \lambda^{\prime}\right\rangle+ \\
1 / Q\left|i, \lambda ; i+2, \lambda^{\prime}\right\rangle+ \\
\sum_{\tilde{\lambda} \tilde{\lambda}^{\prime}} \sum_{\mu}\left\langle\tilde{\lambda}^{\prime}\left|\tilde{P}^{\mu}\right| \lambda\right\rangle\left\langle\tilde{\lambda}\left|\tilde{P}^{\mu}\right| \lambda^{\prime}\right\rangle\left|i, \tilde{\lambda}^{\prime} ; i+1, \tilde{\lambda}\right\rangle .
\end{array}
$$

Substituting this to the two particle Schrödinger equation leads to a similar constraint for the coefficients as in the ferromagnetic case:

$$
\begin{aligned}
& \sum_{\tilde{\lambda}, \tilde{\lambda^{\prime}}}\left[\delta_{\lambda}^{\tilde{\lambda}} \delta_{\lambda^{\prime}}^{\tilde{\lambda}^{\prime}}+\frac{1}{e^{i k^{\prime}}+e^{-i k}} M_{\lambda \lambda^{\prime}}^{\tilde{\lambda} \tilde{\lambda}^{\prime}}\right] B_{\tilde{\lambda}^{\prime}, \tilde{\lambda}}= \\
& -\sum_{\tilde{\lambda}, \tilde{\lambda}^{\prime}}\left[\delta_{\lambda}^{\tilde{\lambda}} \delta_{\lambda^{\prime}}^{\tilde{\lambda}^{\prime}}+\frac{1}{e^{i k}+e^{-i k^{\prime}}} M_{\lambda \lambda^{\prime}}^{\tilde{\lambda} \tilde{\lambda}^{\prime}}\right] A_{\tilde{\lambda}, \tilde{\lambda^{\prime}}},
\end{aligned}
$$

where

$$
M_{\lambda \lambda^{\prime}}^{\tilde{\lambda} \tilde{\lambda}^{\prime}}=\sum_{\mu}\left\langle\tilde{\lambda}^{\prime}\left|\tilde{P}^{\mu}\right| \lambda\right\rangle\left\langle\tilde{\lambda}\left|\tilde{P}^{\mu}\right| \lambda^{\prime}\right\rangle
$$

Similar to the ferromagnetic case, in the limit of vanishing quasiparticle momenta $k, k^{\prime} \rightarrow 0$, this equation can be solved to obtain the $\mathcal{S}$-matrix for $Q=3$ :

$$
\mathcal{S}_{\lambda, \lambda^{\prime}}^{\tilde{\lambda}, \tilde{\lambda}^{\prime}}\left(k, k^{\prime} \rightarrow 0\right)=(-1) \delta_{\lambda}^{\tilde{\lambda}^{\prime}} \delta_{\lambda^{\prime}}^{\tilde{\lambda}} .
$$

\section{APPENDIX B: COMPUTATION OF THE RELAXATION FUNCTIONS ASSUMING A DIAGONAL $\mathcal{S}$-MATRIX FOR $Q=3$}

\section{1. case: Ferromagnetic side}

Let us compute the correlation function $C_{\mu \mu^{\prime}}^{\mathrm{ferro}}(x, t)$ defined as the probability that the domain containing $(0,0)$ has the orientation $\mu$ while that at $(x, t)$ has orientation $\mu^{\prime}$. Note that $C_{\mu \mu^{\prime}}$ is defined in terms of the $P^{\mu}$ 's rather than the $\tilde{P}^{\mu}$ 's.

We can calculate this probability by just keeping track of the number $N_{+}$and $N_{-}$of kink excitations (domain walls) with quantum numbers + and - , respectively, that cross the line between the points $(0,0)$ and $(x, t)$. The points $(0,0)$ and $(x, t)$ have the same domain orientation if $N_{+}$equals $N_{-}$up to modulo three.

Thus the correlation function is

$$
\begin{gathered}
C_{\mu \mu^{\prime}}^{\mathrm{ferro}}(x, t)=\frac{1}{3} \sum_{n} P_{n}[(0,0) \rightarrow(x, t)] \times \\
\times \sum_{k}\left(\begin{array}{l}
n \\
k
\end{array}\right) \frac{1}{2^{n}} \hat{\delta}(2 k-n+\Delta \mu),
\end{gathered}
$$

where $P_{n}[(0,0) \rightarrow(x, t)]$ is the probability that the line $(0,0) \rightarrow(x, t)$ cuts precisely $n$ domain walls, $k$ is the number of upsteps, $\hat{\delta}(m)$ is the discrete delta function modulo 3 , and $\Delta \mu=\mu^{\prime}-\mu$. The probability that we cut 
the path of $n$ particles when moving from $(0,0)$ to $(x, t)$ can be computed in terms of cutting none:

$$
P_{n}((0,0) \rightarrow(x, t))=\left(\begin{array}{c}
M \\
n
\end{array}\right) \bar{P}^{M-n}(1-\bar{P})^{n},
$$

where $M$ is the total number of particles and

$$
\begin{gathered}
\bar{P}=P\left(\text { will not cut } \nu^{\prime} \text { th }\right)=\int_{-L / 2}^{0} \frac{d x_{\nu}}{L} \int_{-\infty}^{\frac{x-x_{\nu}}{t}} d v_{\nu} P\left(v_{\nu}\right) \\
+\int_{0}^{L / 2} \frac{d x_{\nu}}{L} \int_{\frac{x-x_{\nu}}{t}}^{\infty} d v_{\nu} P\left(v_{\nu}\right) .
\end{gathered}
$$

which can be calculated to get

$$
P\left(\text { will not cut } \nu^{\prime} t h\right)=1-\frac{\bar{t}}{M}\left[\frac{1}{\sqrt{\pi}} e^{-u^{2}}+u \operatorname{erf}(u)\right] \text {, }
$$

with $u$ the dimensionless velocity introduced earlier, $u=$ $\bar{x} / \bar{t}$.

Using then the representation of the delta function

$$
\hat{\delta}(m)=\operatorname{Re}\left[\frac{2}{3} e^{i \frac{2 \pi}{3} m}\right]+\frac{1}{3}
$$

all summations above can be evaluated to yield

$$
C_{\mu \mu^{\prime}}^{\mathrm{ferro}}(x, t)=\frac{1}{9}+\frac{1}{3}\left(\delta_{\mu \mu^{\prime}}-\frac{1}{3}\right) e^{-\frac{3}{2} \bar{t} G(u)},
$$

leading to the relaxation function given in the main text, Eq. (24).

\section{2. case: Paramagnetic side}

Two differences arise when we use the diagonal $\mathcal{S}$ matrix, Eq. (11). The first one is that the sequence of the colors now does change in course of the scattering process, while the quantum number of a given line does not. The second change is that the number of the $(-1)$ factors picked up by the wave function now depends on the quantum numbers of two colliding particles. This second change implies that, after averaging over the color of the other quasiparticles, one gets identically 0 if the particle created by the operator $\tilde{P}^{\mu}$ collides with any other particle. Thus the relaxation function is simply the probability that the created particle propagating from point $(0,0)$ to $(x, t)$ collides with no other particle.

The probability that the $\nu$ 'th particle will not collide with the injected one is given by Eq. (B4).

Thus the probability that the injected particle does not collide with any of the already existing quasiparticles simply reads

$$
\begin{gathered}
R_{\text {integrable }}^{\text {para }}(\bar{x}, \bar{t})=\prod_{\nu} P\left(\text { will not cut } \nu^{\prime} t h\right)= \\
=\left(1-\frac{\bar{t} G(u)}{M}\right)^{M} \rightarrow e^{-\bar{t} G(u)}
\end{gathered}
$$

resulting in the relaxation function given by Eq (23).

\section{APPENDIX C: RELATION BETWEEN THE EFFETIVE HAMILTONIAN AND THE SCATTERING MATRIX}

Already simple renormalization group arguments suggest that the $\mathcal{S}$-matrices above describe the scattering of quasiparticles with vanishing momenta for any coupling $g \neq g_{c}$ and $T \ll \Delta$ : We know very well that the coupling $g$ is relevant for $g>g_{c}$ and scales to $g \rightarrow \infty$ under the RG flow, while for $g<g_{c}$ it is irrelevant and scales to $g \rightarrow 0$. The asymptotic (long wavelength) dynamical properties, however, remain invariant under the renormalization group, and therefore the scattering matrix of quasiparticles with vanishing momenta obtained by simply performing perturbation theory in $1 / g$ (or $g$ ) around the trivial fixed point Hamiltonians must coincide with the exact scattering in both phases.

One can refine the renormalization group argument above to show that, apart from some very special points, the $\mathcal{S}$-matrix of the quantum Potts model on a lattice should always take on the simple form, Eq. (10) for vanishing quasiparticle momenta as follows. Let us suppose that we have a high energy cut-off $\Lambda$, larger than the gap, $\Lambda>\Delta$. Performing a renormalization group transformation down to a length scale $b \gg \Delta^{-1}$, we obtain a local Hamiltonian for the elementary excitations ('dressed' local flips and kinks for $g>g_{c}$ and $g<g_{c}$, respectively), that we can simply construct based upon symmetry considerations and power counting:

$$
\begin{aligned}
H_{\mathrm{eff}} & =-\sum_{i} \frac{c^{2}}{2 \Delta} \frac{\partial}{\partial x_{i}{ }^{2}}+\sum_{i<j} u_{\lambda_{i}, \lambda_{j}}^{\lambda_{i}^{\prime}, \lambda_{j}^{\prime}} \delta\left(x_{i}-x_{j}\right) \\
& + \text { irrelevant terms }
\end{aligned}
$$

where $x_{i}$ denotes the coordinate of the $i$ 'th quasiparticle. Here the second interaction term is always relevant, and it is this term that determines the structure of the $\mathcal{S}$ matrix for vanishing momenta. For the sake of simplicity, let us restrict ourselves to the most interesting $Q=3$ case. Then $\lambda$ is just a chirality label, $\lambda= \pm$, and the interaction matrix $u_{\lambda_{i}, \lambda_{j}}^{\lambda_{j}^{\prime}, \lambda_{j}^{\prime}}$ can be characterized through three dimensionless parameters, $u_{1} \equiv u_{++}^{++}=u_{--}^{--}, u_{2} \equiv$ $u_{+-}^{+-}=u_{-+}^{-+}$, and $u_{3} \equiv u_{-+}^{+-}=u_{+-}^{-+}$. These parameters are dimensionless functions of $\Delta b$ and $\Delta / \Lambda$ and can be written as

$$
u_{\alpha}=u_{\alpha}\left(\Delta b, \frac{\Delta}{\Lambda}\right), \quad \alpha=1,2,3 .
$$

In the $b \rightarrow \infty$ (large wavelength) limit these must scale to constants that only depend on the ratio $\Delta / \Lambda$,

$$
\lim _{b \rightarrow \infty} u_{\alpha}\left(\Delta b, \frac{\Delta}{\Lambda}\right)=u_{\alpha}\left(\frac{\Lambda}{\Delta}\right) \text {. }
$$

These three numbers determine then the asymptotic form of the $\mathcal{S}$-matrix. It is then a simple matter to show that 
the $\mathcal{S}$-matrix generated by this effective interaction always assumes the form Eq. [10 ${ }^{43}$ excepting the special case when

$$
u_{2}^{2}=u_{3}^{2} .
$$

In this latter case the interaction is singular (vanishes in one of the scattering channels) and one obtains an $\mathcal{S}$-matrix that is diagonal for vanishing quasiparticle momenta, as given by Eq. (11). This $\mathcal{S}$-matrix coincides with the one that one obtains by requiring integrability, i.e. by requring that the scattering matrices satisfy the Yang-Baxter relations ${ }^{44}$ Thus Eq. (C4) can be viewed as an integrability condiction. However, the $Q=3$ state Potts model is not integrable away from the critical point.

Although there is no obvious reason for that, nevertheless, it might be possible that Eq. (C4) is satisfied if one removes the cut-off from the theory, i.e. if one takes the limite $\Lambda / \Delta \rightarrow \infty$. Indeed, the scattering matrix (11) is only obtained in a continuum field theory ap- proach, when one removes the cut-off and also assumes integrability 31.44 .45 .

However, in a theory with a cut-off there is not a single reason why the integrability condition, Eq. (C4), should be satisfied. In fact, our perturbative expansion just proves that Eq. (C4) is not satisfied in the large $g$ and small $g$ limits.

These arguments very convincingly support that the $\mathcal{S}$-matrix of the quantum Potts model on a lattice always takes on the the form (10) in the limit of vanishing momenta, and the low energy fixed point theory is simply generically not integrable ${ }^{43}$ It is an interesting question if there exists still a cross-over regime for $\Delta \ll \Lambda$, i.e., if there is some regime where for intermediate energies $\Delta \ll c^{2} q^{2} / \Delta \sim T>T^{*}$, the diagonal $\mathcal{S}$-matrix is adequate, but the discussion of this requires extensive numerical studies and is certainly beyond the scope of the present paper $\underline{\underline{43}}$
1 S. Sachdev, Quantum Phase Transitions, (Cambridge University Press, 1999).

${ }^{2}$ For a review, see S. L. Sondhi, S. M. Girvin, J. P. Carini and D. Shahar, Rev. Mod. Phys. 69, 315 (1997).

3 B. Andraka and A. M. Tsvelik, Phys. Rev. Lett. 67, 2886 (1991).

4 A. Schroeder, G. Aeppli, E. Bucher, R. Ramazashvili and P. Coleman, Phys. Rev. Lett. 80, 5623 (1998).

5 M. C. Aronson, M. B. Maple, P. DeSa, A. M. Tsvelick and R. Osborn, Europhys. Lett. 40, 245 (1997); M. C. Aronson, M. B. Maple, R. Chau, A. Georges, A. M. Tsvelik and R. Osborn, J. Phys. Condens. Matter 8, 9815 (1996); M. C. Aronson, R. Osborn, R. A. Robinson, J. W. Lynn, R. Chau, C. L. Seaman and M. B. Maple, Phys. Rev. Lett. 75, 725 (1995).

6 J.-G. Park, D. T. Adroja, K. A. McEwen and A. P. Murani, J. Phys. Condens. Matter 14, 3865 (2002); M. B. Maple, R. P. Dickey, J. Herrmann, M. C. de Andrade, E. J. Freeman, D. A. Gajewski and R. Chau, J. Phys. Condens. Matter 8, 9773 (1996); S. D. Wilson, P. Dai, D. T. Adroja S.-H. Lee, J.-H. Chung, J. W. Lynn, N. P. Butch and M. B. Maple, Phys. Rev. Lett. 94, 056402 (2005); E. D. Bauer, V. S. Zapf, P.-C. Ho, N. P. Butch, E. J. Freeman, C. Sirvent, and M. B. Maple Phys. Rev. Lett. 94, 046401 (2005).

7 C. M. Varma, P. B. Littlewood, S. Schmittrink, E. Abrahams, and A. E. Ruckenstein, Phys. Rev. Lett. 63, 1996 (1989); C. M. Varma, Phys. Rev. Lett. 83, 3538 (1999).

8 S. Sachdev, Science 288, 475 (2000); S. Sachdev, Rev. Mod. Phys. 75, 913 (2003).

${ }^{9}$ N. D. Mathur, F. M. Grosche, S. R. Julian, I. R. Walker,D. M. Freye, R. K. W. Haselwimmer and G. G. Lonzarich, Nature (London) 394, 39 (1998).

10 A. J. Millis, Phys. Rev. B 48, 7183 (1993).

11 A. Georges, O. Parcollet, and S. Sachdev Phys. Rev. B 63, 134406 (2001); A. Georges, O. Parcollet, and S. Sachdev Phys. Rev. Lett. 85, 840-843 (2000).

12 S. Sachdev and P. Young, Phys. Rev. Lett. 78, 2220 (1997).

13 D. Controzzi, F. H. L. Essler, and A. M. Tsvelik, Phys.
Rev. Lett. 86, 680 (2001).

14 J. Karadamoglou, X. Zotos, Phys. Rev. Lett. 93, 177203 (2004).

15 G. Chaboussant, M.-H. Julien, Y. Fagot-Revurat, L. P. Levy, C. Berthier, M. Horvatic, and O. Piovesana, Phys. Rev. Lett. 79, 925 (1997); M. Azuma, Z. Hiroi, M. Takano, K. Ishida, and Y. Kitaoka Phys. Rev. Lett. 73, 3463 (1994); A. V. Sologubenko, S. M. Kazakov, and H. H. Ott, Phys. Rev. B 68, 094432 (2003); G. Xu, J. F. DiTusa, T. Ito, K. Oka, H. Takagi, C. Broholm, and G. Aeppli, Phys. Rev. B 54, R6827 (1996); M. Takigawa, T. Asano, Y. Ajiro, M. Mekata, and Y. J. Uemura, Phys. Rev. Lett. 76, 2173 (1996).

16 D. Bitko, T. F. Rosenbaum, G. Aeppli, Phys. Rev. Lett. 77, 940, (1996).

17 L. Onsager, Phys. Rev. 65, 117 (1944); for a review on the field theory of the critical point see e.g., C. Itzykson and J.-M. Drouffe, Statistical Field Theory I-II, (Cambridge Univ. Press, 1989).

18 P. Di Francesco, P. Mathieu, D. Sénéchal, Conformal Field Theory, (Springer-Verlag, 1997).

19 R. B. Potts, Proc. Cambridge Phil. Soc. 48, 106 (1952).

${ }^{20}$ F. Y. Wu, Rev. Mod. Phys. 54, 235 (1982).

21 V. S. Dotsenko and V. A. Fateev, Nucl. Phys. B 240, 312 (1984).

22 L. Mittag and M. J. Stephen, J. Math. Phys. 12, 441 (1971).

23 J. Sólyom and P. Pfeuty, Phys. Rev. B 24, 218 (1981); J. Sólyom, Phys. Rev. B 24, 230 (1981).

24 J. V. Jose and L. P. Kadanoff, S. Kirkpatrick, and D. R. Nelson, Phys. Rev. B 15, 1217 (1977).

25 J. Salas, A. D. Sokal, J. Stat Phys. 88, 567 (1997); R. H. Swendsen, D. Andelman and A. Nihat Berker, Phys. Rev. B 24, 6732 (1981); J. L. Cardy, M. Nauenberg, and D. J. Scalapino, Phys. Rev. B 22, 2560-2568 (1980).

26 K. Damle and S. Sachdev, Phys. Rev. B 57, 8307 (1998).

27 J. Kogut, Rev. Mod. Phys. 51, 659 (1979).

28 J. L. Cardy, Scaling and Renormalization in Statistical 
Physics (Cambridge Univ. Press, 1996).

29 M. P. M. den Nijs, J. Phys. A 12, 1857 (1978).

30 D. W. Jepsen, J. Math. Phys. 6, 405 (1965); J. L. Lebowitz and J. K. Percus, Phys. Rev. 188, 487 (1969).

31 R. Koberle and J.A. Swieca in Phys. Lett. B 86, 209 (1979).

32 F.C. Alcaraz, J. Phys. A, Math. Gen. 23, L1105 (1990). In this paper the mass ratios exctracted numerically from finite size scaling and compared to those predicted by Swieca and Köberle ${ }^{31}$. For the special case, $Q=3$, however, twokink bound states have the same mass as single kinks. As a result, the excitation spectrum can be understood without invoking bound states.

33 See, e.g., F. H. L. Essler and R. M. Konik, From Fields to Strings:Circumnavigating Theoretical Physics, M. Shifman, A. Vainshtein, and J. Wheather (eds), (World Scientific, 2005); cond-mat/0412421

34 D.L. Cox and A. Zawadowski, Adv. Phys. 47, 599 (1998).

35 E. S. Sorensen and I. Affleck, Phys. Rev. B 49, 13235 (1994).

${ }^{36}$ F. D. M. Haldane, Phys. Rev. Lett. 61, 1029 (1988).

37 S. Chakravarty, B. I. Halperin, D. R. Nelson, Phys. Rev.
B 39, 2344 (1989).

38 The $Q>2$ Potts model is believed to be only integrable at the critical point.

39 B. L. Altshuler and A. M. Tsvelik, cond-mat/0505367 This manuscript has later been modified/corrected to treat the case of non-diagonal $\mathcal{S}$-matrices too (B. L. Altshuler, R. M. Konik and A. M. Tsvelik cond-mat/0508618), and a formula similar to ours has been derived by summing up singular terms in a form factor expansion. These results, however, differ from ours and those of Ref. 40, and it is currently not clear what the origin of such a discrepancy may be.

40 K. Damle and S. Sachdev, cond-mat/0507380

41 A. Rosch and N. Andrei, Phys. Rev. Lett. 85, 1092 (2000).

42 P. Lecheminant and E. Orignac, Phys. Rev. B 69, 174409 (2004).

43 A. Rapp, G. Zarand, and U. Schollwöck, unpublished.

44 For a review, see P. Fendley and N. Read, J. Phys. A 35, 10675 (2002).

45 L. Chim and A.B. Zamolodchikov, Int. J. Mod. Phys. A 7, 5317 (1992). 